\title{
CONNECTED SIMPLE SYSTEMS AND THE CONLEY INDEX OF ISOLATED INVARIANT SETS
}

\author{
BY \\ DIETMAR SALAMON ${ }^{1}$ \\ Dedicated to the memory of Charles C. Conley
}

\begin{abstract}
The object of this paper is to present new and simplified proofs for most of the basic results in the index theory for flows. Simple, explicit formulae are derived for the maps which play a central role in the theory. The presentation is self-contained.
\end{abstract}

1. Introduction. Some dynamical processes in physics, chemistry and biology can be described by differential equations depending on parameters which cannot be determined with an arbitrary degree of precision. For the study of such systems it is important to determine those structural properties which remain invariant under (small) perturbations. Many of these properties can be described in terms of an index theory which has been developed by Conley [3]. Although the basic ideas have been developed over many years, complete proofs for some of the central theorems are only recently available (see e.g. Kurland $[6,7,8]$, Conley and Zehnder $[4]$ and Franzosa [5]).

The object of this paper is to present new and simplified proofs for a number of the basic results in the index theory for isolated invariant sets in flows. Furthermore, explicit and simple formulae are derived for those maps which play a central role in the theory, in particular for the identification map between index spaces (formula (4.3)), for the connection map in the coexact sequence (formulae (5.9) and (5.6)) and for the homotopy equivalence in the continuation theorem (formula (6.19)). In previous papers only the existence of these maps has been proved and the available proofs are widely spread in the literature and much more complicated. For the purpose of completeness, this paper gives a self-contained presentation of the basic results in the index theory for flows.

In two preliminary sections we collect some elementary notions and results from homotopy theory (\$2) and from the general theory of flows on topological spaces (§3). In the main part of this paper we introduce the fundamental concepts in the index theory for isolated invariant sets $(\S 4)$, prove the existence and uniqueness of a

Received by the editors December 4, 1984.

1980 Mathematics Subject Classification. Primary 34C35, 54H20, 58F12.

Key words and phrases. Flows, isolated invariant sets, index theory, attractor-repeller pairs, connection map, continuation.

${ }^{1}$ Sponsored by the United States Army under Contract No. DAAG29-80-C-0041. This material is based upon work supported by the National Science Foundation under Grant No. MCS-8210950.

(1985 American Mathematical Society $0002-9947 / 85 \$ 1.00+\$ .25$ per page 
long coexact sequence associated with an attractor-repeller pair (\$5) and establish the basic continuation results for the Conley index ( 6 ).

The starting point for this work was an essential simplification of the proof that any two index pairs for a given isolated invariant set are homotopically equivalent after collapsing the exit set (Lemma 4.7). This result allows a very simple proof of the fact that the Conley index of an isolated invariant set is a connected simple system (Lemma 4.8 and Theorem 4.10) and leads to further simplifications in the $\S \S 5$ and 6.

A unique coexact sequence for an attractor-repeller pair can be obtained in two steps. The first step is to show that any index pair can be transformed into an NDR-pair (§5.1) so that general results from homotopy theory can be applied to obtain the existence of a long coexact sequence of index spaces. The second step is then to show that all the maps in this sequence induce morphisms of connected simple systems which are independent of the choice of the index pair (Theorem 5.7).

In §6 we begin with some general results on parametrized local flows $X \times \Lambda$ and in particular we make precise what we mean by a continuous family $\sigma(\lambda)$ of isolated invariant sets in $X \times \lambda(\S 6.1)$. The continuation theorem is then phrased for such a family $\sigma(\lambda)$ and consists of three parts. The first part is to show that the injection map of an index space for $\sigma(\lambda)$ in $X \times \lambda$ into the corresponding index space for the global isolated invariant set $S=\bigcup_{\lambda \in \Lambda} \sigma(\lambda)$ in $X \times \Lambda$ induces a morphism between connected simple systems which is independent of the choice of the index pair (Proposition 6.5). The second step is to show that this injection map is locally a homotopy equivalence and the corresponding morphism of connected simple systems is therefore an isomorphism (Theorem 6.7). This local result allows a global continuation of the Conley index in every compact connected component of the parameter space $\Lambda$ by means of a sequence of compact subsets of $\Lambda$ to each of which the local continuation theorem applies. However, different sequences may lead to different identifications between "far away" index spaces. We show that any two of these connecting equivalences between index spaces are in fact homotopic if $\Lambda$ is simply connected. This means that the "global Conley index" $I(\sigma, X, \Lambda)$ consisting of the index spaces for $\sigma(\lambda)$ in $X \times \lambda$ together with the above connecting equivalences is a connected simple system provided that $\Lambda$ is simply connected (Theorem 6.9). Finally, things are put together to obtain a long coexact sequence for the global Conley index associated with an attractor-repeller pair in the case that $\Lambda$ is simply connected (Theorem 6.10).

2. Homotopy theory. In this preliminary section we recall some basic concepts and results from homotopy theory. We will work in the category of pointed topological spaces and continuous, base point preserving maps. In order to avoid unnecessary complications, we will assume that all the spaces under consideration are metrizable and therefore in particular compactly generated. Standard references are Spanier [12] and Whitehead [13].

For any pair $(X, A)$ of topological spaces with $A \subset X$ we denote by

$$
X / A=((X \backslash A) \cup[A],[A])
$$


the pointed space which is obtained by collapsing $A$ to a single point denoted by [ $A$ ]. A set $U \subset X / A$ is open if either $U$ is open in $X$ and $U \cap A=\varnothing$ or the set $(U \cap(X \backslash A)) \cup A$ is open in $X$.

For any two pointed (metric) spaces $\left(X, x_{0}\right)$ and $\left(Y, y_{0}\right)$ the product space $X \times Y$ is understood as a pointed space with base point $\left(x_{0}, y_{0}\right)$. Furthermore, we denote the sum and the smash product of $X$ and $Y$ by

$$
\begin{aligned}
& X \vee Y=X \times y_{0} \cup x_{0} \times Y \subset X \times Y, \\
& X \wedge Y=X \times Y / X \vee Y .
\end{aligned}
$$

For any two maps $f: X \rightarrow X^{\prime}, g: Y \rightarrow Y^{\prime}$ between pointed spaces, the sum $f \vee g$ : $X \vee Y \rightarrow X^{\prime} \vee Y^{\prime}$ and the smash product $f \wedge g: X \wedge Y \rightarrow X^{\prime} \wedge Y^{\prime}$ are defined in an obvious manner. The (unique) constant map between pointed spaces $\left(X, x_{0}\right)$ and $\left(W, w_{0}\right)$ will always be denoted by $c: X \rightarrow W, c(x)=w_{0}$ for all $x \in X$, and the identity map by $1_{x}: X \rightarrow X$ or simply $1: X \rightarrow X$.

The suspension $\Sigma X$ of a pointed space $\left(X, x_{0}\right)$ is defined by

$$
\Sigma X=X \times[0,1] / X \times 0 \cup x_{0} \times[0,1] \cup X \times 1 \text {. }
$$

Hence $\Sigma X=X \wedge \Sigma^{1}$, where $\Sigma^{1}=[0,1] /\{0,1\}$ is the pointed circle. The pointed $n$-sphere is given by $\Sigma^{n}=\Sigma \Sigma^{n-1}=\Sigma^{1} \wedge \cdots \wedge \Sigma^{1}$ ( $n$ times). The suspension $\Sigma \varphi$ of a map $\varphi: X \rightarrow Y$ between pointed spaces is defined by $\Sigma \varphi=\varphi \wedge 1: \Sigma X \rightarrow \Sigma Y$.

For any two pointed spaces $\left(X, x_{0}\right)$ and $\left(W, w_{0}\right)$ we denote by $[X ; W]$ the set of homotopy classes of continuous, base point preserving maps from $X$ to $W$. Then $[X ; W]$ is a pointed set, the distinguished point being the class of the constant map. We denote by $[f]$ the homotopy class of the continuous, base point preserving map $f: X \rightarrow W$ and by $[X]$ the homotopy type of the pointed space $X$. If two maps $f_{0}: X \rightarrow W$ and $f_{1}: X \rightarrow W$ are homotopic we denote this by $f_{0} \sim f_{1}$.

For any two pointed spaces $\left(X, x_{0}\right),\left(Y, y_{0}\right)$ and any continuous, base point preserving map $\varphi: X \rightarrow Y$ the mapping cone $T_{\varphi}$ is given by

$$
T_{\varphi}=X \times[0,1] \cup_{\varphi} Y / X \times 0 \cup x_{0} \times[0,1],
$$

where the topological space $X \times[0,1] \cup_{\varphi} Y$ is obtained from the disjoint union of $X \times[0,1]$ and $Y$ by identifying the pair $(x, 1) \in X \times[0,1]$ with $\varphi(x) \in Y$ for every $x \in X$. Note that there is a natural injection of $Y$ into $T_{\varphi}$. The importance of this concept is based upon the following simple observation.

Remark 2.1. Let $\varphi: X \rightarrow Y$ and $g: Y \rightarrow W$ be continuous, base point preserving maps between pointed spaces. Then $g \circ \varphi \sim c: X \rightarrow W$ if and only if the following lifting problem has a solution:

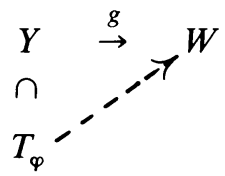

Now let $\left(X, x_{0}\right),\left(Y, y_{0}\right),\left(W, w_{0}\right)$ be pointed spaces and let $\varphi: X \rightarrow Y$ be a continuous, base point preserving map. Then the induced map

$$
\varphi^{\#}:[Y ; W] \rightarrow[X ; W]
$$


is defined by $\varphi^{\#}[g]=[g \circ \varphi]$ for $[g] \in[Y ; W]$. The sequence

$$
X \stackrel{\varphi}{\rightarrow} Y \stackrel{\psi}{\rightarrow} Z
$$

of continous, base point preserving maps between pointed spaces is said to be coexact if for every pointed space $\left(W, w_{0}\right)$ the induced sequence

$$
[X ; W] \stackrel{\varphi^{*}}{\leftarrow}[Y ; W] \stackrel{\psi^{*}}{\leftarrow}[Z ; W]
$$

is exact. This means that $\operatorname{ker} \varphi^{\#}=$ range $\psi^{\#}$, where

$$
\begin{gathered}
\operatorname{ker} \varphi^{\#}=\{[g] \in[Y ; W] \mid g \circ \varphi \sim c: X \rightarrow W\}, \\
\operatorname{range} \psi^{\#}=\{[h \circ \psi] \in[Y ; W] \mid[h] \in[Z ; W]\} .
\end{gathered}
$$

Choosing $W=Z$ and $h=1_{Z}$ or, respectively, $W=T_{\varphi}$ and $g=j: Y \rightarrow T_{\varphi}$ the canonical injection, we obtain the following useful characterization for the sequence (2.1) to be coexact.

Proposition 2.2. The sequence (2.1) is coexact if and only if the following two conditions are satisfied.

(i) $\psi \circ \varphi \sim c: X \rightarrow Z$, i.e. the lifting problem

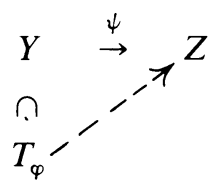

has a solution.

(ii) There exists a map $h: Z \rightarrow T_{\varphi}$ such that $h \circ \psi \sim j: Y \rightarrow T_{\varphi}, j$ being the canonical injection of $Y$ into $T_{\varphi}$.

Now let the pointed space $\left(X, x_{0}\right)$ be a closed subspace of $\left(Y, x_{0}\right)$, let $\iota: X \rightarrow Y$ denote the canonical injection and $\pi: Y \rightarrow Y / X$ the canonical projection map, and consider the sequence

$$
X \stackrel{\iota}{\rightarrow} Y \stackrel{\pi}{\rightarrow} Y / X .
$$

Then $\pi \circ \iota=c: X \rightarrow Y / X$. Hence it follows from Proposition 2.2 that a sufficient condition for the sequence (2.2) to be coexact is that the map $\iota: X \rightarrow Y$ is a cofibration in the sense that the lifting problem

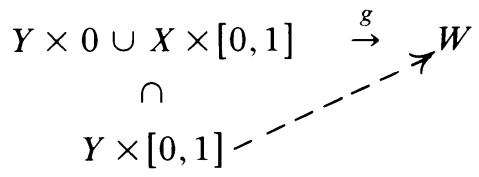

has a solution for every topological space $W$ and every continuous map $g: Y \times 0 \cup X \times[0,1] \rightarrow W$. Equivalently, $(X, Y)$ is an NDR-pair in the following sense. 
DEFINITION 2.3 (NDR - PAIR). Let $X$ be a closed subset of the metric space $Y$. Then $(X, Y)$ is said to be an NDR-pair if there exist continuous maps $r: Y \times[0,1] \rightarrow Y$ and $\alpha: Y \rightarrow[0,1]$ such that

1. $\alpha(y)=0 \Leftrightarrow y \in X$,

2. $r(y, 0)=y \forall y \in Y$,

3. $r(x, \sigma)=x \forall x \in X, \forall \sigma \in[0,1]$,

4. $r(y, 1) \in X \forall y \in Y$ with $\alpha(y)<1$.

In fact, if $X, Y$ is an NDR-pair and the continuous maps $r: Y \times[0,1] \rightarrow Y$ and $\alpha: Y \rightarrow[0,1]$ satisfy the conditions of Definition 2.3, then the map

$$
G: Y \times[0,1] \rightarrow Y \times 0 \cup X \times[0,1],
$$

defined by

$$
G(y, \sigma)= \begin{cases}(r(y, 1), \sigma-2 \alpha(y)), & 0 \leqslant \alpha(y) \leqslant \sigma / 2, \\ (r(y, 2-2 \alpha(y) / \sigma), 0), & \sigma / 2<\alpha(y)<\sigma, \\ (y, 0), & \sigma \leqslant \alpha(y) \leqslant 1,\end{cases}
$$

for $y \in Y$ and $\sigma \in[0,1]$, is continuous and satisfies $G(y, 0)=(y, 0)$ and $G(x, \sigma)=$ $(x, \sigma)$ for all $y \in Y, x \in X, \sigma \in[0,1]$. Therefore we have the following result.

Proposition 2.4. Let $\left(X, x_{0}\right)$ be a closed subspace of the pointed metric space $\left(Y, x_{0}\right)$. Then the following statements are equivalent.

(i) $\iota: X \rightarrow Y$ is a cofibration.

(ii) There exists a continuous map $G: Y \times[0,1] \rightarrow Y \times 0 \cup X \times[0,1]$ such that $G(y, 0)=(y, 0)$ and $G(x, \sigma)=(x, \sigma)$ for all $y \in Y, x \in X, \sigma \in[0,1]$.

(iii) $X, Y$ is an NDR-pair.

If these conditions are satisfied, then the sequence (2.2) is coexact.

The map $G: Y \times[0,1] \rightarrow Y \times 0 \cup X \times[0,1]$ defined by (2.4) gives rise to a connection map $\delta: Y / X \rightarrow \Sigma X$ which leads to a long coexact sequence. More precisely, we have the following important result (see e.g. Whitehead [13, Theorem III.6.22]).

THEOREM 2.5. Let $\left(X, x_{0}\right)$ be a closed subspace of the pointed metric space $\left(Y, x_{0}\right)$, suppose that $X, Y$ is an NDR-pair and let the maps $r: Y \times[0,1] \rightarrow Y$ and $\alpha$ : $Y \rightarrow[0,1]$ satisfy the conditions of Definition 2.3. Let the connection map $\delta: Y / X \rightarrow \Sigma X$ be defined by

$$
\delta([y])= \begin{cases}(r(y, 1), 1-2 \alpha(y)), & 0<\alpha(y)<1 / 2, \\ {[X \times 0],} & \text { otherwise }\end{cases}
$$

for $y \in Y$. Then the sequence

$$
X \stackrel{\iota}{\rightarrow} Y \stackrel{\pi}{\rightarrow} Y / X \stackrel{\delta}{\rightarrow} \Sigma X \stackrel{\Sigma_{\iota}}{\rightarrow} \Sigma Y \stackrel{\Sigma \pi}{\rightarrow} \Sigma Y / X \stackrel{\Sigma \delta}{\rightarrow} \ldots
$$

is coexact. 
Proof. Let $p_{X}: Y \times 0 \cup X \times[0,1] \rightarrow \Sigma X$ be the natural projection which collapses $Y \times 0 \cup x_{0} \times[0,1] \cup X \times 1$ to a single point. Then $p_{X} \circ G$ provides a homotopy between $c: Y \rightarrow \Sigma X$ and $\delta \circ \pi: Y \rightarrow \Sigma X$. Now let the maps

$$
\begin{gathered}
j: \Sigma X \rightarrow T_{\pi}=Y \times[0,1] / Y \times 0 \cup x_{0} \times[0,1] \cup X \times 1, \\
k: Y / X \rightarrow T_{\pi}, \quad k([y])=[y, 1],
\end{gathered}
$$

be the natural injections. Then $j \circ \delta \sim k: Y / X \rightarrow T_{\pi}$ via the homotopy

$$
H: Y / X \times[0,1] \rightarrow T_{\pi}
$$

which is defined by

$$
H([y], \sigma)= \begin{cases}{[r(y, 1), 1-2 \alpha(y)],} & 0 \leqslant \alpha(y) \leqslant \sigma / 2 \\ {[r(y, 2-2 \alpha(y) / \sigma), 1-\sigma],} & \sigma / 2<\alpha(y)<\sigma \\ {[y, 1-\sigma],} & \sigma \leqslant \alpha(y) \leqslant 1\end{cases}
$$

Hence it follows from Proposition 2.2 that the sequence $Y \stackrel{\pi}{\rightarrow} Y / X \stackrel{\delta}{\rightarrow} \Sigma X$ is coexact.

Now let $p_{Y}: T_{\pi} \rightarrow \Sigma Y$ be the natural projection which collapses $Y \times 1$. Then the map $p_{Y} \circ H: Y / X \times[0,1] \rightarrow \Sigma Y$ provides a homotopy between $c: Y / X \rightarrow \Sigma Y$ and $\Sigma \iota \circ \delta: Y / X \rightarrow \Sigma Y$. Finally let us define the map

$$
d: \Sigma Y \rightarrow T_{\delta}=Y / X \times[0,1] \cup_{\delta} \Sigma X /[X] \times[0,1] \cup Y / X \times 0
$$

by

$$
d([y, \sigma])= \begin{cases}{[[y], 2-2 \sigma] \in Y / X \times[0,1],} & 1 / 2 \leqslant \sigma \leqslant 1, \\ {[r(y, 1), 2 \sigma-2 \alpha(y)] \in \Sigma X,} & 0 \leqslant \alpha(y) \leqslant \sigma \leqslant 1 / 2, \\ {[Y / X \times 0],} & \sigma \leqslant \alpha(y) \leqslant 1,0 \leqslant \sigma \leqslant 1 / 2,\end{cases}
$$

for $[y, \sigma] \in \Sigma Y$. Then the map $d \circ \Sigma \iota: \Sigma X \rightarrow T_{\delta}$ is given by

$$
d \circ \Sigma_{\iota}([x, \sigma])= \begin{cases}{[x, 2 \sigma],} & 0 \leqslant \sigma \leqslant 1 / 2, \\ {[Y / X \times 0],} & \text { otherwise }\end{cases}
$$

for $[x, \sigma] \in \Sigma X$. This map is homotopic to the canonical injection $l: \Sigma X \rightarrow T_{\delta}$ via the homotopy $\Phi: \Sigma X \times[0,1] \rightarrow T_{\delta}$ which is given by

$$
\Phi([x, \sigma], \tau)= \begin{cases}{[x,(1+\tau) \sigma],} & 0 \leqslant(1+\tau) \sigma \leqslant 1, \\ {[Y / X \times 0],} & \text { otherwise }\end{cases}
$$

for $[x, \sigma] \in \Sigma X$ and $\tau \in[0,1]$. Hence it follows from Proposition 2.2 that the sequence

$$
Y / X \stackrel{\delta}{\rightarrow} \Sigma X \stackrel{\Sigma_{\iota}}{\rightarrow} \Sigma Y
$$

is coexact.

Finally, Proposition 2.2 shows that if any sequence of the form (2.1) is coexact, then so is the sequence

$$
\Sigma X \stackrel{\Sigma \varphi}{\rightarrow} \Sigma Y \stackrel{\Sigma \psi}{\rightarrow} \Sigma Z
$$

of the suspensions. 
At the end of this section we introduce the extremely useful concept of a connected simple system which is due to Conley [3]. A connected simple system is a subcategory of the category of pointed spaces and homotopy classes of maps between these with the additional property that for any two objects there is a unique morphism between these (in each direction). More precisely, we make the following definition.

DEFINITION 2.6 (CONNECTED SIMPLE SYSTEM). A connected simple system consists of a collection $I_{0}$ of pointed spaces along with a collection $I_{m}$ of homotopy classes of maps between these such that

(i) $\operatorname{hom}(X, \bar{X})=\left\{[f] \in[X ; \bar{X}] \mid[f] \in I_{m}\right\}$ is nonempty and consists of a single element for each ordered pair $X, \bar{X}$ of spaces in $I_{0}$,

(ii) if $X, \bar{X}, \overline{\bar{X}} \in I_{0}$ and $[f] \in \operatorname{hom}(X, \bar{X}),[\bar{f}] \in \operatorname{hom}(\bar{X}, \overline{\bar{X}})$, then $[\bar{f} \circ f] \in$ $\operatorname{hom}(X, \overline{\bar{X}})$,

(iii) $\operatorname{hom}(X, X)=\left\{\left[1_{X}\right]\right\}$ for all $X \in I_{0}$.

Note that each morphism in a connected simple system is necessarily the homotopy class of a homotopy equivalence. Morphisms between connected simple systems are defined as follows.

Definition 2.7. A morphism $\Phi: I \rightarrow J$ between the connected simple systems $I=\left(I_{0}, I_{m}\right)$ and $J=\left(J_{0}, J_{m}\right)$ is a collection of homotopy classes of maps between spaces in $I_{0}$ and spaces in $J_{0}$ such that

(i) for every $X \in I_{0}$ and every $Y \in J_{0}$ the $\operatorname{set} \Phi(X, Y)=\{[\varphi] \in[X ; Y] \mid[\varphi] \in \Phi\}$ is nonempty and consists of a single element,

(ii) if $X, \bar{X} \in I_{0}$ and $Y, \bar{Y} \in J_{0}$ and if $[\varphi] \in \Phi(X, Y),[f] \in \operatorname{hom}(\bar{X}, X),[g] \in$ $\operatorname{hom}(Y, \bar{Y})$, then $[g \circ \varphi \circ f] \in \Phi(\bar{X}, \bar{Y})$.

Of course, any single map $\varphi: X \rightarrow Y, X \in I_{0}, Y \in J_{0}$, induces a morphism between the connected simple systems $I$ and $J$ via property (ii) in the above definition. If a morphism $\Phi: I \rightarrow J$ consists of homotopy equivalences, then the homotopy inverses of these maps induce a morphism $\Phi^{-1}: J \rightarrow I$. Finally we mention that the suspension functor $\Sigma$ associates with any connected simple system $I=\left(I_{0}, I_{m}\right)$ the connected simple system $\Sigma I=\left(\Sigma I_{0}, \Sigma I_{m}\right)$ which is defined by

$$
\Sigma I_{0}=\left\{\Sigma X \mid X \in I_{0}\right\}, \quad \Sigma I_{m}=\left\{[\Sigma f] \mid[f] \in I_{m}\right\}
$$

3. Flows. In this section we collect some elementary properties of flows. Although the results are known we indicate at some places the main ideas of the proofs. Basic references are Birkhoff [2], Bhatia and Szegö [1] and Conley [3].

Let $\Gamma$ be a Hausdorff topological space and let the continuous map $(\gamma, t) \rightarrow \gamma \cdot t$ from $\Gamma \times \mathbf{R}$ into $\Gamma$ be a flow, that is $\gamma \cdot 0=\gamma$ and $\gamma \cdot(t+s)=(\gamma \cdot t) \cdot s$ for every $\gamma \in \Gamma$ and all $t, s \in \mathbf{R}$.

We point out that the Hausdorff property for the flow $\Gamma$ is too strong for some dynamical systems. In particular the space of curves $\gamma: I \rightarrow X$ from an open interval $I \subset \mathbf{R}$ into a metric space $X$ is not Hausdorff in the compact-open topology, even if one considers only those curves whose graph is closed in $\mathbf{R} \times X$. The whole theory can also be developed in the more general situation that $\Gamma$ is not a Hausdorff space, but this causes some technical difficulties such as the definition of $\omega$-limit sets relative to a subset $X \subset \Gamma$. In order to avoid these difficulties and to make the main 
ideas more transparent, we restrict ourselves to the case that $\Gamma$ is a Hausdorff space.

A set $S \subset \Gamma$ is said to be invariant if $S \cdot \mathbf{R}=S$. The maximal invariant subset of a set $N \subset \Gamma$ is given by

$$
I(N)=\{\gamma \in \Gamma \mid \gamma \cdot \mathbf{R} \subset N\} .
$$

If $N$ is closed then so is $I(N)$, since the closure of any invariant set is invariant. The $\omega$-limit sets of a set $Y \subset \Gamma$ are given by

$$
\begin{gathered}
\omega(Y)=I(\operatorname{cl}(Y \cdot[0, \infty)))=\bigcap_{t>0} \operatorname{cl}(Y \cdot[t, \infty)), \\
\omega^{*}(Y)=I(\operatorname{cl}(Y \cdot(-\infty, 0]))=\bigcap_{t>0} \operatorname{cl}(Y \cdot(-\infty,-t]) .
\end{gathered}
$$

Now let $S \subset \Gamma$ be a compact invariant set and let $Y \subset S$. Then $\omega(Y)$ and $\omega^{*}(Y)$ are compact invariant subsets of $S$ and they are connected if $Y$ is. Furthermore, if $U$ is a neighborhood of $\omega(Y)$, then there exists a $t>0$ such that $Y \cdot[t, \infty) \subset U$. A similar statement holds for $\omega^{*}(Y)$. A compact invariant set $A \subset S$ is said to be an attractor in $S$ if there exists a neighborhood $U$ of $A$ in $S$ such that $A=\omega(U)$. A compact invariant set $A^{*} \subset S$ is said to be a repeller in $S$ if there exists a neighborhood $U$ of $A^{*}$ in $S$ such that $A^{*}=\omega^{*}(U)$. The following lemma gives a very useful characterization of attractors.

Lemma 3.1. Let $S \subset \Gamma$ be a compact invariant set. Then a compact invariant set $A \subset S$ is an attractor in $S$ if and only if there exists a neighborhood $U$ of $A$ in $S$ such that $\gamma \cdot(-\infty, 0] \not \subset U$ for all $\gamma \in U \backslash A$.

Proof. The necessity of the condition is clear since $\gamma \cdot(-\infty, 0] \subset U$ implies $\gamma \in \omega(U)$. If $U$ is a compact neighborhood of $A$ in $S$ such that $\gamma \cdot(-\infty, 0] \not \subset U$ for all $\gamma \in U \backslash A$, then there exists a $t^{*}>0$ such that $\gamma \cdot\left[-t^{*}, 0\right] \not \subset U$ for all $\gamma \in U \cap$ $\operatorname{cl}(S \backslash U)$. Now choose a neighborhood $V$ of $A$ such that $V \cdot\left[0, t^{*}\right] \subset U$. Then $V \cdot[0, \infty) \subset U$ and therefore $\omega(V)=A$.

Lemma 3.2. Let $S \subset \Gamma$ be a compact invariant set and let $A$ be an attractor in $S$. Then the following statements hold.

(i) If $\gamma \in S$ and $\omega^{*}(\gamma) \cap A \neq \varnothing$, then $\gamma \in A$.

(ii) If $\gamma \in S$ and $\omega(\gamma) \cap A \neq \varnothing$, then $\omega(\gamma) \subset A$.

(iii) $A^{*}=\{\gamma \in S \mid \omega(\gamma) \cap A=\varnothing\}$ is a repeller in $S$, called the complementary repeller of $A$.

(iv) $A=\left\{\gamma \in S \mid \omega^{*}(\gamma) \cap A^{*}=\varnothing\right\}$.

(v) If $V$ is a compact neighborhood of $A$ in $S$ with $V \cap A^{*}=\varnothing$, then $A=\omega(V)$.

(vi) If $\gamma \in S$, then $\omega(\gamma) \cup \omega^{*}(\gamma) \subset A \cup A^{*}$.

(vii) If $A^{\prime}$ is an attractor in $A$, then $A^{\prime}$ is an attractor in $S$.

Proof. Let $U$ be an open neighborhood of $A$ in $S$ such that $\omega(U)=A$.

(i) If $\omega^{*}(\gamma) \cap A \neq \varnothing$, then $\gamma \cdot\left(-t_{n}\right) \in U$ for some sequence $t_{n}$ tending to $\infty$ and hence $\gamma \in \omega(U)=A$.

(ii) If $\omega(\gamma) \cap A \neq \varnothing$, then $\gamma \cdot t \in U$ for some $t \geqslant 0$ and therefore $\omega(\gamma)=$ $\omega(\gamma \cdot t) \subset \omega(U)=A$. 
(iii) Choose $t^{*}>0$ such that $\operatorname{cl}\left(U \cdot\left[t^{*}, \infty\right)\right) \subset U$ and define

$$
U^{*}=S \backslash \operatorname{cl}\left(U \cdot\left[t^{*}, \infty\right)\right) \text {. }
$$

Then $S=U \cup U^{*}$. Furthermore $U^{*} \cdot\left(-\infty,-t^{*}\right] \subset S \backslash U$ and therefore $U^{*}$ is a neighborhood of $\omega^{*}\left(U^{*}\right) \subset S \backslash U \subset U^{*}$. Hence $\omega^{*}\left(U^{*}\right)$ is a repeller in $S$.

If $\gamma \in \omega^{*}\left(U^{*}\right)$, then $\omega(\gamma) \subset \omega^{*}\left(U^{*}\right)$. This implies $\omega(\gamma) \cap A=\varnothing$ and therefore $\gamma \in A^{*}$. If $\gamma \in A^{*}$, then $\gamma \cdot \mathbf{R} \cap U=\varnothing$ since otherwise $\omega(\gamma) \subset \omega(U)=A$. Hence $\gamma \cdot \mathbf{R} \subset U^{*}$ and therefore $\gamma \in I\left(U^{*}\right)=\omega^{*}\left(U^{*}\right)$. We conciude that $A^{*}=\omega^{*}\left(U^{*}\right)$ is a repeller.

(iv) The dual arguments of the preceding ones show that $A=\omega(U)=$ $\left\{\gamma \in S \mid \omega^{*}(\gamma) \cap A^{*}=\varnothing\right\}$.

(v) Let $U^{*}$ be an open neighborhood of $A^{*}$ in $S$ such that $A^{*}=\omega^{*}\left(U^{*}\right)$ and $U^{*} \cap V=\varnothing$. Choose $t^{*}>0$ such that $U^{*} \cdot\left(-\infty,-t^{*}\right] \subset U^{*} \subset S \backslash V$. Then $V \cdot\left[t^{*}, \infty\right) \subset S \backslash U^{*}$ and therefore $\omega(V) \subset S \backslash U^{*}$. By (iv), this implies $\omega(V)=A$.

(vi) Follows from (i)-(iv).

(vii) Let $U^{\prime}$ be a neighborhood of $A^{\prime}$ in $S$ such that $U^{\prime} \subset U$ and $\omega\left(U^{\prime} \cap A\right)=A^{\prime}$. Let $\gamma \in U^{\prime}$ such that $\gamma \cdot(-\infty, 0] \subset U^{\prime}$. Then $\gamma \cdot(-\infty, 0] \subset U$ and therefore $\gamma \in \omega(U)=A$. Hence $\gamma \cdot(-\infty, 0] \subset U^{\prime} \cap A$ and therefore $\gamma \in \omega\left(U^{\prime} \cap A\right)=A^{\prime}$. By Lemma 3.1, this implies that $A^{\prime}$ is an attractor in $S$.

Let $A_{1}$ and $A_{2}$ be attractors in a compact, invariant set $S \subset \Gamma$. Then it follows from Lemma 3.1 that $A_{1} \cap A_{2}$ is an attractor in $S$ and from Lemma 3.2(iii) that $A_{1}^{*} \cup A_{2}^{*}$ is its complementary repeller. By duality, $A_{1} \cup A_{2}$ is an attractor in $S$ and $A_{1}^{*} \cap A_{2}^{*}$ is its complementary repeller.

We are now going to introduce the concept of a Morse decomposition of an invariant set $S$. This concept serves as a tool to generalize the classical Morse theory for gradient flows on compact manifolds with finitely many critical points to arbitrary flows and isolated invariant sets. One of the essential features of the general approach is the continuation theorem which cannot be derived in the context of the classical theory. Also, in the classical theory there are no means to define an index for invariant sets other than critical points such as periodic solutions or invariant tori.

DEFINITION 3.3 (MORSE DECOMPOSITION). Let $S \subset \Gamma$ be a compact, invariant set. Then a finite collection $\{M(\pi) \mid \pi \in P\}$ of compact invariant sets in $S$ is said to be a Morse decomposition of $S$ if there exists an ordering $\pi_{1}, \ldots, \pi_{n}$ of $P$ such that for every $\gamma \in S \backslash \cup_{\pi \in P} M(\pi)$ there exist indices $i, j \in\{1, \ldots, n\}$ such that $i<j$ and

$$
\omega(\gamma) \subset M\left(\pi_{i}\right), \quad \omega^{*}(\gamma) \subset M\left(\pi_{j}\right) .
$$

Every ordering of $P$ with this property is said to be admissible. The sets $M(\pi)$ are called Morse sets.

If $S$ is a compact, invariant set in $\Gamma$ and $\{M(\pi) \mid \pi \in P\}$ a Morse decomposition of $S$, then for $\pi, \pi^{*} \in P$ we define $\pi<\pi^{*}$ if $\pi \neq \pi^{*}$ and $\pi$ comes before $\pi^{*}$ in every admissible ordering of $P$. This defines a partial order on $P$. Clearly, any total ordering of $P$ is admissible if and only if it preserves the partial order on $P$. A subset $I \subset P$ is said to be an interval if

$$
\pi^{\prime}, \pi^{\prime \prime} \in I, \quad \pi \in P, \quad \pi^{\prime}<\pi<\pi^{\prime \prime} \Rightarrow \pi \in I .
$$


For any interval $I$ we define the set

$$
M(I)=\left\{\gamma \in S \mid \omega(\gamma) \cup \omega^{*}(\gamma) \subset \bigcup_{\pi \in I} M(\pi)\right\} .
$$

In the following proposition we collect some basic properties of the partially ordered set $(P,<)$.

Proposition 3.4. Let $S \subset \Gamma$ be a compact invariant set and let $\{M(\pi) \mid \pi \in P\}$ be a Morse decomposition of $S$ with the associated partial order " $<$ " on $P$. Then the following statements hold.

(i) If $I \subset P$ is an interval, then there exists an admissible ordering $\pi_{1}, \ldots, \pi_{n}$ of $P$ and $i, j \in\{1, \ldots, n\}, i \leqslant j$, such that $I=\left\{\pi_{i}, \ldots, \pi_{j}\right\}$.

(ii) If $\left\{\pi, \pi^{*}\right\} \subset P$ is an interval, then $\pi<\pi^{*}$ if and only if there exists a $\gamma \in S$ such that $\omega(\gamma) \subset M(\pi)$ and $\omega^{*}(\gamma) \subset M\left(\pi^{*}\right)$.

(iii) Let $\pi, \pi^{*} \in P$. Then $\pi<\pi^{*}$ if and only if there exist sequences $\pi_{0}=\pi$, $\pi_{1}, \ldots, \pi_{k}=\pi^{*} \in P$ and $\gamma_{1}, \ldots, \gamma_{k} \in S \backslash \bigcup\{M(\pi) \mid \pi \in P\}$ such that

$$
\omega\left(\gamma_{j}\right) \subset M\left(\pi_{j-1}\right), \quad \omega^{*}\left(\gamma_{j}\right) \subset M\left(\pi_{j}\right), \quad j=1, \ldots, k .
$$

(iv) Let $I \subset P$ be an interval. Then $M(I)$ is an attractor in $S$ if and only if

$$
\pi^{\prime} \in P, \quad \pi \in I, \quad \pi^{\prime}<\pi \Rightarrow \pi^{\prime} \in I .
$$

In this case $M(P \backslash I)$ is the complementary repeller of $M(I)$ in $S$ and $I$ is said to be an attractor interval and $P \backslash I$ a repeller interval.

(v) If $I \subset P$ is an interval, then $M(I)$ is a compact invariant set, $\{M(\pi) \mid \pi \in I\}$ is a Morse decomposition of $M(I)$ and $\{M(\pi) \mid \pi \in P \backslash I\} \cup\{M(I)\}$ is a Morse decomposition of $S$.

Proof. (i) Let $I \subset P$ be an interval. Then the sets $J=\left\{\pi \in P \mid \exists \pi^{\prime} \in I\right.$ with $\left.\pi<\pi^{\prime}\right\}$ and $K=P \backslash(I \cup J)$ are intervals and can be ordered in the form $J, I, K$, preserving the partial order on $P$. Now choose an ordering of $P$ which preserves the ordering of the sets $J, I, K$ and the partial order on $P$.

(ii) Let $\left\{\pi, \pi^{*}\right\}$ be an interval and suppose that $\pi<\pi^{*}$. Then, by (i), there exists an admissible ordering on $P$ such that $\pi^{*}$ follows immediately on $\pi$. Hence there exists a $\gamma \in S$ with $\omega(\gamma) \subset M(\pi)$ and $\omega^{*}(\gamma) \subset \mathrm{M}\left(\pi^{*}\right)$, since otherwise one would get another admissible ordering by interchanging $\pi$ and $\pi^{*}$, contradicting $\pi<\pi^{*}$.

(iii) Suppose that $\pi<\pi^{*}$ and construct a sequence $\pi_{0}=\pi<\pi_{1}<\cdots<\pi_{k}=\pi^{*}$ such that there is no $\pi \in P$ and no $j \in\{1, \ldots, k\}$ with $\pi_{j-1}<\pi<\pi_{j}$. Then the sets $\left\{\pi_{j-1}, \pi_{j}\right\} \subset P$ are intervals and therefore (iii) follows from (ii).

(iv) If $I \subset P$ does not satisfy (3.1), then there exist $\pi \in P \backslash I, \pi^{*} \in I$ such that $\left\{\pi, \pi^{*}\right\}$ is an interval and $\pi<\pi^{*}$. Hence it follows from (ii) that $M(I)$ cannot be an attractor.

In order to prove the converse implication, let $\pi^{*} \in P$ satisfy

$$
\pi^{*} \nless \pi \quad \forall \pi \in P .
$$

Then $I=P \backslash\left\{\pi^{*}\right\}$ is an interval with the property (3.1). Let $U^{*}$ be a neighborhood of $M\left(\pi^{*}\right)$ in $S$ with $\operatorname{cl}\left(U^{*}\right) \cap M(\pi)=\varnothing$ for all $\pi \in I$ and let $\gamma \in U^{*} \backslash M\left(\pi^{*}\right)$. 
Then it follows from (iii) and (3.2) that $\omega(\gamma) \cap \operatorname{cl}\left(U^{*}\right)=\varnothing$ and therefore $\gamma \cdot[0, \infty) \not \subset U^{*}$. Hence the dual result of Lemma 3.1 shows that $M\left(\pi^{*}\right)$ is a repeller in $S$. The complementary attractor of $M\left(\pi^{*}\right)$ is given by $M(I)=\{\gamma \in$ $\left.S \mid \omega^{*}(\gamma) \not \subset M\left(\pi^{*}\right)\right\}$. Therefore $M(I)$ is an attractor in $S$ and in particular a compact invariant set. Now statement (iv) follows by induction with $S$ replaced by $M(I)$.

Statement (v) is a direct consequence of (iv) and (i).

The notion of an attractor-interval as well as the proof of the previous proposition are due to Franzosa [5].

The following concept has turned out to be very useful for the development of a theory which covers a wide range of applications.

Definition 3.5 (LOCAL FlOW). A locally compact subset $X \subset \Gamma$ is said to be a local flow if for every $\gamma \in X$ there exists a neighborhood $U$ of $\gamma$ in $\Gamma$ and an $\varepsilon>0$ such that $(X \cap U) \cdot[0, \varepsilon) \subset X$.

Whenever $Y \subset X$ we denote from now on by $\operatorname{cl}(Y)$ its closure relative to $X$ and by $\operatorname{int}(Y)$ its interior relative to $X$.

DEFINITION 3.6 (ISOlATED INVARIANT SET). Let $X \subset \Gamma$ be a local flow and let $S \subset X$ be a compact invariant set. Then $S$ is said to be an isolated invariant set if there exists a compact neighborhood $N$ of $S$ in $X$ such that $S=I(N)$. In that case $N$ is said to be an isolating neighborhood (for $S$ in $X$ ).

If $N_{1}$ and $N_{2}$ are isolating neighborhoods for the isolated invariant sets $S_{1}$ and $S_{2}$, respectively, in the local flow $X \subset \Gamma$, then $S_{1} \cap S_{2}$ is an isolated invariant set in $X$ with the isolating neighborhood $N_{1} \cap N_{2}$. Figure 1 shows that there is no corresponding statement for the union of isolated invariant sets.

LEMMA 3.7. If $N$ is an isolating neighborhood for the isolated invariant set $S$ in the metric local flow $X \subset \Gamma$, then there exists a neighborhood $\bar{N}$ of $N$ in $X$ which is still an isolating neighborhood for $S$.

Proof. If the statement were false, then there would exist a sequence $\gamma_{k} \in X \backslash N$ such that $d\left(\gamma_{k} \cdot t, N\right) \leqslant 1 / k$ for all $k \in \mathbf{N}$ and $t \in \mathbf{R}$. A limit point $\gamma$ of $\gamma_{k}$ would then satisfy $\gamma \in \operatorname{cl}(X \backslash N) \cap I(N)$, a contradiction.

If $S$ is an isolated invariant set in a local flow $X \subset \Gamma$ and $\{M(\pi) \mid \pi \in P\}$ is a Morse decomposition of $S$, then the Morse sets $M(\pi)$ are also isolated invariant sets in $X$.

The following compactness result has been established in Conley and Zehnder [4, Lemma 3.1]. For the sake of completeness we present a slightly simplified proof.

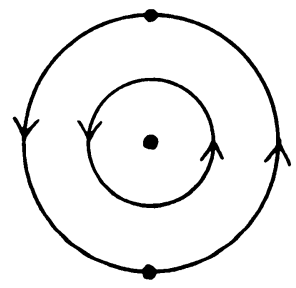

FIGURE 1 
LEMMA 3.8. Let $N \subset X$ be an isolating neighborhood for the isolated invariant set $S$ in the metric local flow $X \subset \Gamma$, let $\{M(\pi) \mid \pi \in P\}$ be a Morse decomposition of $S$ and let " $<$ " be the associated partial order on $P$. Then the following statements hold.

(i) If $\gamma \cdot[0, \infty) \subset N$, then there is a $\pi \in P$ with $\omega(\gamma) \subset M(\pi)$. If $\gamma \cdot(-\infty, 0] \subset$ $N$, then there is a $\pi^{*} \in P$ with $\omega^{*}(\gamma) \subset M\left(\pi^{*}\right)$.

(ii) If $I \subset P$ is an attractor interval, then the sets

$$
\begin{gathered}
M^{-}(I)=\left\{\gamma \in N \mid \gamma \cdot(-\infty, 0] \subset N \text { and } \omega^{*}(\gamma) \subset M(\pi) \text { for some } \pi \in I\right\}, \\
M^{+}(P \backslash I)=\{\gamma \in N \mid \gamma \cdot[0, \infty) \subset N \text { and } \omega(\gamma) \subset M(\pi) \text { for some } \pi \in P \backslash I\}
\end{gathered}
$$

are compact.

Proof. Proposition 3.4 allows us to reduce both statements to the case $P=$ $\left\{\pi, \pi^{*}\right\}$, where $\{\pi\}$ is an attractor interval, that is $\pi^{*} \nless \pi$.

In order to prove statement (i), let us assume that $\gamma \cdot[0, \infty) \subset N$ and $\omega(\gamma) \not \subset$ $M(\pi)$ and $\omega(\gamma) \not \subset M\left(\pi^{*}\right)$. Since $\omega(\gamma)$ is connected, this implies that $\omega(\gamma) \not \subset M(\pi)$ $\cup M\left(\pi^{*}\right)$ and hence there exists a $\gamma^{\prime} \in \omega(\gamma) \subset S$ with $\gamma^{\prime} \notin M(\pi) \cup M\left(\pi^{*}\right)$. Therefore $\omega\left(\gamma^{\prime}\right) \subset M(\pi)$ and $\omega^{*}\left(\gamma^{\prime}\right) \subset M\left(\pi^{*}\right)$. Making use of the fact that $\gamma^{\prime} \cdot \mathbf{R} \subset \omega(\gamma)$, we obtain that $M(\pi) \cap \omega(\gamma) \neq \varnothing$ and $M\left(\pi^{*}\right) \cap \omega(\gamma) \neq \varnothing$. Now let us choose an open neighborhood $U$ of $M(\pi)$ in $N$ such that $\operatorname{cl}(U) \cap M\left(\pi^{*}\right)=\varnothing$. Then there exists a sequence $t_{n} \geqslant 0$ tending to infinity such that $\gamma \cdot t_{n} \in U, \gamma_{0}=\lim _{n \rightarrow \infty} \gamma \cdot t_{n}$ $\in M(\pi)$ and $\gamma \cdot\left[t_{n}, t_{n+1}\right] \not \subset U$. Hence there exists a sequence $t_{n}^{\prime} \in\left[t_{n}, t_{n+1}\right]$ with $\gamma \cdot\left[t_{n}, t_{n}^{\prime}\right] \subset \operatorname{cl}(U)$ and $\gamma \cdot t_{n}^{\prime} \notin U$. Let $\gamma_{1}$ be any limit point of $\gamma \cdot t_{n}^{\prime}$. Then $\gamma_{1} \in$ $N \backslash U$ and $\gamma_{1} \in \omega(\gamma) \subset S$. Furthermore the sequence $t_{n}^{\prime}-t_{n}$ has to be unbounded since otherwise $\gamma_{1} \in \gamma_{0} \cdot \mathbf{R} \subset M(\pi)$. But this implies that $\gamma_{1} \cdot(-\infty, 0] \subset \operatorname{cl}(U)$ and therefore $\omega^{*}\left(\gamma_{1}\right) \subset I(U)=M(\pi)$. Since $\gamma_{1} \notin M(\pi)$ we conclude that $\omega\left(\gamma_{1}\right) \subset$ $M\left(\pi^{*}\right)$, contradicting the fact that $\pi^{*} \nless \pi$. This proves the first assertion in (i). The second assertion in (i) can be established by analogous arguments.

For statement (ii) it is enough to show that the respective subsets of $N$ are closed since $N$ is a compact Hausdorff space.

$M^{+}(P)$ is closed: Let $\gamma \in N \backslash M^{+}(P)$. Then by (i), there exists a $t>0$ such that $\gamma \cdot t \notin N$. This implies that $\gamma^{\prime} \cdot t \notin N$ for all $\gamma^{\prime}$ in some open neighborhood of $\gamma$ in $N$. Hence $N \backslash M^{+}(P)$ is open in $N$ and therefore $M^{+}(P)$ is closed.

$M^{+}\left(\pi^{*}\right)$ is closed: Let $\gamma_{n} \in M^{+}\left(\pi^{*}\right) \subset M^{+}(P)$ converge to $\gamma \in M^{+}(P)$ and suppose that $\gamma \notin M^{+}\left(\pi^{*}\right)$. Then $\omega(\gamma) \subset M(\pi)$ and $\omega\left(\gamma_{n}\right) \subset M\left(\pi^{*}\right)$ for all $n \in \mathbf{N}$. Now let $U$ be an open neighborhood of $M\left(\pi^{*}\right)$ in $X$ such that $\operatorname{cl}(U) \cap M(\pi)=\varnothing$. Since $\omega(\gamma) \subset M(\pi)$, there exists a $t>0$ such that $\gamma \cdot t \notin \operatorname{cl}(U)$ and therefore $\gamma_{n} \cdot t \notin \operatorname{cl}(U)$ for every sufficiently large $n \in \mathbf{N}$. For each of these $n \in \mathbf{N}$ there exists a $t_{n}>0$ such that $\gamma_{n} \cdot\left(t_{n}, \infty\right) \subset U \cap N$ and $\gamma_{n} \cdot t_{n} \notin U$. Let us choose a subsequence such that $\gamma_{n} \cdot t_{n}$ converges to $\gamma^{*}$. Then $\gamma^{*} \notin U$ and $\gamma^{*} \cdot[0, \infty) \subset \operatorname{cl}(U) \cap N$ which implies that $\omega\left(\gamma^{*}\right) \subset M\left(\pi^{*}\right)$. Moreover, the sequence $t_{n}$ has to be unbounded since otherwise $\gamma^{*} \in \gamma \cdot \mathbf{R}$ and thus $\omega\left(\gamma^{*}\right) \subset M(\pi)$. Hence we obtain that $\gamma^{*} \cdot t=\lim _{n \rightarrow \infty} \gamma_{n}$. $\left(t_{n}+t\right) \in N$ for all $t \in \mathbf{R}$ and thus $\gamma^{*} \in S$. Recalling that $\gamma^{*} \notin U$, we conclude that $\omega^{*}\left(\gamma^{*}\right) \not \subset M\left(\pi^{*}\right)$ and therefore $\omega^{*}\left(\gamma^{*}\right) \subset M(\pi)$. But this is a contradiction to $\pi^{*} \nless \pi$. 
The closedness of $M^{-}(P)$ and $M^{-}(\pi)$ can be established by analogous arguments.

At the end of this section we establish an important consequence of the local flow property.

LEMmA 3.9. Let $X \subset \Gamma$ be a local flow and suppose that $\gamma \cdot[0, T] \subset X$. Furthermore, let $W$ be a neighborhood of $\gamma \cdot[0, T]$ in $X$. Then there exists a neighborhood $U$ of $\gamma$ in $\Gamma$ such that $(U \cap X) \cdot[0, T] \subset W$.

Proof. Let $W_{0}$ be a compact neighborhood of $\gamma \cdot[0, T]$ in $X$ and define $V=W$ $\cap W_{0}$. Then $\partial V=\operatorname{cl}(V) \cap \operatorname{cl}(X \backslash V)$ is a compact set. Therefore $\partial V$ is closed in $\Gamma$ and $\gamma \cdot[0, T] \cap \partial V=\varnothing$. Hence it follows from the continuity of the flow together with the compactness of $[0, T]$ that there is a neighborhood $U$ of $\gamma$ in $\Gamma$ such that

$$
U \cap X \subset V, \quad(U \cap X) \cdot[0, T] \subset \Gamma \backslash \partial V .
$$

Now let $\gamma^{\prime} \in U \cap X$ and suppose that $\gamma^{\prime} \cdot[0, T] \not \subset V$. Then

$$
t^{\prime}=\sup \left\{t>0 \mid \gamma^{\prime} \cdot[0, t] \subset V\right\} \in[0, T]
$$

and $\gamma^{\prime} \cdot t^{\prime} \in \operatorname{cl}(V) \subset X$. Since $X$ is a local flow, there exists an $\varepsilon>0$ such that $\gamma^{\prime} \cdot\left[t^{\prime}, t^{\prime}+\varepsilon\right) \subset X$. But the definition of $t^{\prime}$ implies that $\gamma^{\prime} \cdot\left[t^{\prime}, t^{\prime}+\delta\right) \not \subset V$ for any $\delta>0$ and therefore $\gamma^{\prime} \cdot t^{\prime} \in \partial V$. This contradicts the definition of $U$. We conclude that $(U \cap X) \cdot[0, T] \subset V \subset W$.

\section{Index theory.}

4.1. Existence of index pairs. The concept of an index pair plays a crucial role in the definition of the Conley index for isolated invariant sets. For the introduction of this concept we need the notion of positive invariance. Let $N$ be a compact subset of a local flow $X \subset \Gamma$. Then a subset $K \subset N$ is said to be positively invariant in $N$ if

$$
\gamma \in K, \quad t \geqslant 0, \quad \gamma \cdot[0, t] \subset N \Rightarrow \gamma \cdot t \in K .
$$

Definition 4.1 (INDEX PAIRS). Let $X \subset \Gamma$ be a local flow and let $S \subset X$ be an isolated invariant set. Then a pair $\left(N_{1}, N_{0}\right)$ of compact sets in $X$ is said to be an index pair for $S$ in $X$ if $N_{0} \subset N_{1}$ and

(i) $N_{1} \backslash N_{0}$ is a neighborhood of $S$ in $X$ and $S=I\left(\mathrm{cl}\left(N_{1} \backslash N_{0}\right)\right)$,

(ii) $N_{0}$ is positively invariant in $N_{1}$, and

(iii) if $\gamma \in N_{1}$ and $\gamma \cdot[0, \infty) \not \subset N_{1}$, then there exists a $t \geqslant 0$ with $\gamma \cdot[0, t] \subset N_{1}$ and $\gamma \cdot t \in N_{0}$.

The crucial property (iii) of an index pair $\left(N_{1}, N_{0}\right)$ says that every orbit which leaves $N_{1}$ in forward time has to go through the exit set $N_{0}$ before leaving $N_{1}$.

For any subset $K \subset N$ we define the minimal positively invariant set in $N$ which contains $K$ by

$$
P(K, N)=\{\gamma \in N \mid \exists t \geqslant 0 \text { with } \gamma \cdot[-t, 0] \subset N, \gamma \cdot(-t) \in K\} .
$$

The whole difficulty for proving the existence of index pairs lies in the fact that $P(K, N)$ need not be closed, even if $K$ is closed, and that its closure need not be positively invariant. This is illustrated by Figure 2 in which the (positively invariant) exit set $N^{-}=\{\gamma \in N \mid \gamma \cdot[0, \varepsilon) \not \subset N$ for every $\varepsilon>0\}$ is not closed. 


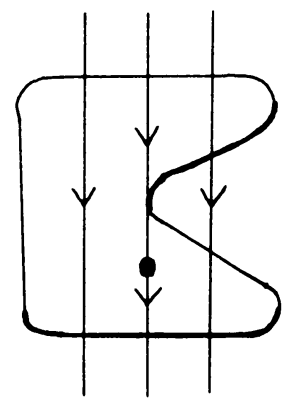

FIGURE 2

This difficulty can be overcome by means of the following lemma which is due to Conley and Zehnder [4, Lemma 3.2]. However, there is a mistake in the proof of this result in [4]. We present a (hopefully) correct proof using an argument in Conley [3, p. 47].

LeMma 4.2. Let $X \subset \Gamma$ be a metric local flow, let $N \subset X$ be an isolating neighborhood of the isolated invariant set $S \subset X$ and let $\{M(\pi) \mid \pi \in P\}$ be a Morse decomposition of $S$ with the associated partial ordering " $<$ " on $P$. Then the following statements hold.

(i) If $K \subset N$ is a compact set with $K \cap M^{+}(P)=\varnothing$, then $P(K, N)$ is compact.

(ii) For every attractor interval $I \subset P$ and every open neighborhood $U$ of $M^{-}(I)$ in $\Gamma$ there exists a compact neighborhood $N_{I}$ of $M^{-}(I)$ in $N$ such that $N_{I} \subset U$ and $N_{I}$ is positively invariant in $N$.

Proof. (i) Let $\gamma_{n} \in P(K, N)$ converge to $\gamma$ and let $t_{n} \geqslant 0$ be chosen such that $\gamma_{n} \cdot\left[-t_{n}, 0\right] \subset N$ and $\gamma_{n} \cdot\left(-t_{n}\right) \in K$. Then the sequence $t_{n}$ has to be bounded since otherwise any limit point $\gamma^{*}$ of $\gamma_{n} \cdot\left(-t_{n}\right)$ satisfies $\gamma^{*} \in K$ and $\gamma^{*} \cdot[0, \infty) \subset N$, contradicting $K \cap M^{+}(P)=\varnothing$. Hence the sequence $t_{n}$ has a limit point $t \geqslant 0$ and we get $\gamma \cdot[-t, 0] \subset N, \gamma \cdot(-t) \in K$, and therefore $\gamma \in P(K, N)$.

(ii) We prove this statement in four steps. Let us first choose any compact neighborhood $W$ of $M(P \backslash I)$ in $N$ such that $W \cap M^{-}(I)=\varnothing$.

Step 1 . If $K \subset N$ is a compact set such that $M^{-}(I) \subset P(K, N) \subset U \cap(N \backslash W)$, then $P(K, N)$ is compact.

Proof. Let $\gamma_{n} \in P(K, N)$ converge to $\gamma$ and choose $t_{n} \geqslant 0$ such that $\gamma_{n} \cdot\left[-t_{n}, 0\right]$ $\subset N$ and $\gamma_{n} \cdot\left(-t_{n}\right) \in K$. Then $\gamma_{n} \cdot\left[-t_{n}, 0\right] \subset P(K, N) \subset U \cap(N \backslash W)$ for all $n \in$ N. If $t_{n}$ is an unbounded sequence, then we obtain $\gamma \cdot(-\infty, 0] \subset \operatorname{cl}(U \cap(N \backslash W))$ which implies $\omega^{*}(\gamma) \subset M(\pi)$ for some $\pi \in I$ and therefore $\gamma \in M^{-}(I) \subset P(K, N)$. If the sequence $t_{n}$ is bounded and $t \geqslant 0$ is a limit point of $t_{n}$, then we conclude that $\gamma \cdot[-t, 0] \subset N, \gamma \cdot(-t) \in K$ and therefore $\gamma \in P(K, N)$.

Step 2. There exists a $t^{*}>0$ such that

$$
\gamma \cdot\left[-t^{*}, 0\right] \subset \operatorname{cl}(N \backslash W) \Rightarrow \gamma \in U \cap(N \backslash W) .
$$


Proof. If this implication would not hold, then there would exist sequences $\gamma_{n} \in N$ and $t_{n} \geqslant 0$ such that $t_{n}$ tends to infinity, $\gamma_{n} \cdot\left[-t_{n}, 0\right] \subset \operatorname{cl}(N \backslash W)$ and $\gamma_{n} \notin U \cap(N \backslash W)$. Any limit point $\gamma$ of $\gamma_{n}$ would then satisfy $\gamma \notin U \cap(N \backslash W)$ and $\gamma \cdot(-\infty, 0] \subset \operatorname{cl}(N \backslash W)$. But this would imply $\omega^{*}(\gamma) \subset M(I)$ and therefore $\gamma \in M^{-}(I) \subset U \cap(N \backslash W)$ which would be a contradiction.

Step 3. Construction of $N_{I}$. Let us define the sets

$$
A=\left\{\gamma \in M^{-}(I) \mid \gamma \cdot\left[0, t^{*}\right] \subset N\right\}, \quad B=\left\{\gamma \in M^{-}(I) \mid \gamma \cdot\left[0, t^{*}\right] \not \subset N\right\} .
$$

Then for every $\gamma \in A$ there exists an open neighborhood $U(\gamma)$ of $\gamma$ in $\Gamma$ such that $U(\gamma) \cdot\left[0, t^{*}\right] \subset U \cap(\Gamma \backslash W)$. For every $\gamma \in B$ there exists a $t(\gamma)>0$ such that $\gamma \cdot[0, t(\gamma)] \subset U \cap(\Gamma \backslash W)$ and $\gamma \cdot t(\gamma) \notin N$ which enables us to choose an open neighborhood $U(\gamma)$ of $\gamma$ in $\Gamma$ such that $U(\gamma) \cdot[0, t(\gamma)] \subset U \cap(\Gamma \backslash W)$ and $U(\gamma) \cdot t(\gamma) \cap N=\varnothing$. Since $M^{-}(I)$ is compact, there exist finitely many $\gamma_{1}, \ldots, \gamma_{k}$ $\in M^{-}(I)$ such that the sets $U\left(\gamma_{j}\right), j=1, \ldots, k$, cover $M^{-}(I)$. We choose a compact neighborhood $K$ of $M^{-}(I)$ in $N$ such that $K \subset \bigcup_{j=1}^{k} U\left(\gamma_{j}\right)$ and define $N_{I}=P(K, N)$.

Step 4. $N_{I} \subset U \cap(N \backslash W)$.

Proof. Let $\gamma \in N_{I}$ and let $t \geqslant 0$ with $\gamma \cdot[-t, 0] \subset N$ and $\gamma \cdot(-t) \in K$. Then $\gamma \cdot(-t) \in U\left(\gamma_{j}\right)$ for some $j \in\{1, \ldots, k\}$. Suppose that $\gamma \notin U \cap(\Gamma \backslash W)$. If $\gamma_{j} \in A$, then $\gamma \cdot\left[-t, t^{*}-t\right] \subset U \cap(\Gamma \backslash W)$ and therefore $t^{*}<t$. Hence there exists a $t^{\prime} \in\left[0, t-t^{*}\right]$ such that $\gamma \cdot\left[-t,-t^{\prime}\right) \subset U \cap(N \backslash W)$ and $\gamma \cdot\left(-t^{\prime}\right) \notin U \cap$ $(N \backslash W)$. This implies $\gamma \cdot\left[-t^{\prime}-t^{*},-t^{\prime}\right] \subset \operatorname{cl}(N \backslash W)$ and $\gamma \cdot\left(-t^{\prime}\right) \notin U \cap(N \backslash W)$, contradicting Step 2. If $\gamma_{j} \in B$, then $\gamma \cdot\left[-t, t\left(\gamma_{j}\right)-t\right] \subset U \cap(\Gamma \backslash W)$ and $\gamma \cdot\left(t\left(\gamma_{j}\right)-t\right) \notin N$. From $\gamma \cdot[-t, 0] \subset N$ we obtain $t\left(\gamma_{j}\right)>t$ and from $\gamma \notin U \cap$ $(\Gamma \backslash W)$ we obtain $t\left(\gamma_{j}\right)<t$, a contradiction. We conclude that $\gamma \in N \cap U \cap$ $(\Gamma \backslash W)=U \cap(N \backslash W)$ which proves Step 4.

By definition, the set $N_{I}$ constructed in Step 3 is a neighborhood of $M^{-}(I)$ in $N$ which is positively invariant in $N$. Furthermore $N_{I}=P(K, N) \subset U \cap(N \backslash W)$ (Step 4) and hence $N_{I}$ is compact (Step 1).

Now we are in the position to establish the following important existence result for index pairs (compare Conley and Zehnder [4, Lemma 3.3]).

THEOREM 4.3 (EXISTENCE OF INDEX PAIRS). Let $X \subset \Gamma$ be a metric local flow, let $N \subset X$ be an isolating neighborhood of the isolated invariant set $S \subset X$ and let $U$ be any neighborhood of $S$ in $\Gamma$. Then there exists an index pair $\left(N_{1}, N_{0}\right)$ for $S$ in $X$ such that $N_{1}$ and $N_{0}$ are positively invariant in $N$ and $\operatorname{cl}\left(N_{1} \backslash N_{0}\right) \subset U$.

Proof. By Lemma 3.7, the sets

$$
M^{+}=\{\gamma \in N \mid \gamma \cdot[0, \infty) \subset N\}, \quad M^{-}=\{\gamma \in N \mid \gamma \cdot(-\infty, 0] \subset N\}
$$

are compact and $S=M^{+} \cap M^{-}$. Hence there exist open neighborhoods $U^{+}$of $M^{+}$in $N$ and $U^{-}$of $M^{-}$in $N$ such that

$$
\operatorname{cl}\left(U^{+} \cap U^{-}\right) \subset U \cap \text { int } N .
$$

By Lemma 4.2 there exists a compact, positively invariant set $N_{1}^{\prime}$ in $N$ such that $N_{1}^{\prime} \subset U^{-}$and $N_{1}^{\prime}$ is a neighborhood of $M^{-}$in $N$. Now define

$$
N_{0}=P\left(N \backslash U^{+}, N\right), \quad N_{1}=N_{1}^{\prime} \cup N_{0} .
$$


Then $N_{0}$ and $N_{1}$ are positively invariant in $N$ and $N_{1} \backslash N_{0} \subset U^{+} \cap U^{-}$and hence $\operatorname{cl}\left(N_{1} \backslash N_{0}\right) \subset U$. Furthermore $N_{0}$ is compact (Lemma 4.2(i)) and $N_{0} \cap S=\varnothing$. Therefore $N_{1} \backslash N_{0}$ is a neighborhood of $S$. Clearly $S=I\left(\operatorname{cl}\left(N_{1} \backslash N_{0}\right)\right)$, since $S \subset$ $\operatorname{cl}\left(N_{1} \backslash N_{0}\right) \subset N$. It remains to show that $N_{0}$ is the exit set of $N_{1}$.

For this purpose let $\gamma \in N_{1}$ such that $\gamma \cdot[0, \infty) \not \subset N_{1}$ and suppose that $\gamma \notin N_{0}$. Define $t^{*}=\sup \left\{t>0 \mid \gamma \cdot[0, t] \subset N_{1} \backslash N_{0}\right\}$. Then

$$
\gamma \cdot t^{*} \in \operatorname{cl}\left(N_{1} \backslash N_{0}\right) \subset \operatorname{cl}\left(U^{+} \cap U^{-}\right) \subset \operatorname{int} N .
$$

Since $X$ is a local flow there exists an $\varepsilon>0$ such that $\gamma \cdot\left[t^{*}, t^{*}+\varepsilon\right] \subset$ int $N$. Since $\gamma \cdot t^{*} \in N_{1}$ we conclude that $\gamma \cdot\left[t^{*}, t^{*}+\varepsilon\right] \subset N_{1}$ and $\gamma \cdot\left[t^{*}, t^{*}+\varepsilon\right] \cap N_{0} \neq \varnothing$.

The next result shows the existence of a filtration of index pairs associated with a Morse decomposition of $S$ (compare Conley and Zehnder [4, Theorem 3.1]).

Corollary 4.4. Let $X \subset \Gamma$ be a metric local flow, let $S \subset X$ be an isolated invariant set and let $\{M(\pi), \pi \in P\}$ be a Morse decomposition of $S$ with an admissible ordering $\pi_{1}, \ldots, \pi_{n}$ of $P$. Furthermore let $\left(N_{n}, N_{0}\right)$ be an index pair for $S$ in $X$. Then there exists a filtration $N_{0} \subset N_{1} \subset \cdots \subset N_{n-1} \subset N_{n}$ of compact sets such that $\left(N_{k}, N_{j-1}\right)$ is an index pair for

$$
M_{k j}=\left\{\gamma \in S \mid \omega(\gamma) \cup \omega^{*}(\gamma) \subset \bigcup_{i=j}^{k} M\left(\pi_{i}\right)\right\}
$$

whenever $1 \leqslant j \leqslant k \leqslant n$.

Proof. Define $N=\operatorname{cl}\left(N_{n} \backslash N_{0}\right)$. Then, by Lemma 3.7, for any $j \in\{1, \ldots, n\}$ the sets

$$
\begin{aligned}
& M_{j}^{+}=\left\{\gamma \in N \mid \gamma \cdot[0, \infty) \subset N, \omega(\gamma) \subset M_{n j}\right\}, \\
& M_{j}^{-}=\left\{\gamma \in N \mid \gamma \cdot(-\infty, 0] \subset N, \omega^{*}(\gamma) \subset M_{j 1}\right\}
\end{aligned}
$$

are compact. Now let $N_{n}^{\prime}=N \cap N_{n}$ and define $N_{j}^{\prime} \subset N$ recursively such that $N_{j}^{\prime}$ is a compact neighborhood of $M_{j}^{-}$in $N$ which is positively invariant in $N_{j+1}^{\prime}$ and satisfies $N_{j}^{\prime} \cap M_{j+1}^{+}=\varnothing\left(\right.$ Lemma 4.2), $j=n-1, \ldots, 1$. Then the sets $N_{j}=N_{j}^{\prime} \cup N_{0}$ satisfy the requirements of the corollary.

REMARK 4.5. A very nice refinement of the previous result has recently been established by Franzosa [5]. Let $N \subset X$ be an isolating neighborhood of the isolated invariant set $S$ in the metric local flow $X \subset \Gamma$ and let $\{M(\pi) \mid \pi \in P\}$ be a Morse decomposition of $S$ with the associated partial order " $<$ " on $P$. Let $\mathscr{J}$ denote the set of attractor intervals in $P$. Then Franzosa has shown in [5] that there exists a family $\{N(I) \mid I \in \mathscr{J}\}$ of compact, positively invariant sets in $N$ such that

(i) $(N(J), N(I))$ is an index pair for $M(J \backslash I)$ for all $I, J \in \mathscr{J}$ with $I \subset J$, and

(ii) $N(I \cap J)=N(I) \cap N(J), N(I \cup J)=N(I) \cup N(J)$ for all $I, J \in \mathscr{J}$.

The proof is not easy. The essential difficulty is the requirement that both the intersection and the union property have to be satisfied simultaneously.

4.2. Equivalence of index pairs. The most important property of index pairs is that the homotopy type of the pointed space $N_{1} / N_{0}$ is independent of the choice of the index pair and therefore depends only on the behavior of the flow near the isolated 
invariant set $S$ (Conley and Zehnder [4] and Kurland [6]). We present a highly simplified proof of this fact. More precisely, we will show that for any isolated invariant set $S$ in a local flow $X$ the collection

$$
\left\{N_{1} / N_{0} \mid\left(N_{1}, N_{0}\right) \text { is an index pair for } S \text { in } X\right\}
$$

along with a certain collection of homotopy classes of flow induced maps is a connected simple system in the sense of Definition 2.6. The proof consists of the following three lemmas.

LEMMA 4.6. Let $N$ be an isolating neighborhood for the isolated invariant set $S$ in the metric local flow $X \subset \Gamma$ and let $U$ be a neighborhood of $S$ in $X$. Then there exists a $t>0$ such that $\gamma \cdot[-t, t] \subset N \Rightarrow \gamma \in U$.

Proof. If there would exist sequences $\gamma_{k} \in X \backslash U$ and $t_{k} \geqslant 0$ such that $t_{k}$ tends to infinity and $\gamma_{k} \cdot\left[-t_{k}, t_{k}\right] \subset N$ for all $k \in \mathbf{N}$, then any limit point $\gamma$ of $\gamma_{k}$ would satisfy $\gamma \in \operatorname{cl}(X \backslash U)$ and $\gamma \cdot \mathbf{R} \subset N$. This would imply $\gamma \in S \cap \operatorname{cl}(X \backslash U)$, a contradiction.

The next lemma defines a flow induced map from $N_{1} / N_{0}$ into $\bar{N}_{1} / \bar{N}_{0}$ for any two index pairs $\left(N_{1}, N_{0}\right),\left(\bar{N}_{1}, \bar{N}_{0}\right)$ of $S$ in $X$.

Lemma 4.7. Let $\left(N_{1}, N_{0}\right)$ and $\left(\bar{N}_{1}, \bar{N}_{0}\right)$ be index pairs for the isolated invariant set $S$ and choose $T \geqslant 0$ such that the following implications hold for $t \geqslant T$ :

$$
\begin{aligned}
& \gamma \cdot[-t, t] \subset N_{1} \backslash N_{0} \Rightarrow \gamma \in \bar{N}_{1} \backslash \bar{N}_{0}, \\
& \gamma \cdot[-t, t] \subset \bar{N}_{1} \backslash \bar{N}_{0} \Rightarrow \gamma \in N_{1} \backslash N_{0} .
\end{aligned}
$$

Then the map $f: N_{1} / N_{0} \times[T, \infty) \rightarrow \bar{N}_{1} / \bar{N}_{0}$ defined by

$$
f([\gamma], t)=f^{t}([\gamma])= \begin{cases}{[\gamma \cdot 3 t],} & \text { if } \gamma \cdot[0,2 t] \subset N_{1} \backslash N_{0}, \gamma \cdot[t, 3 t] \subset \bar{N}_{1} \backslash \bar{N}_{0}, \\ {\left[\bar{N}_{0}\right],} & \text { otherwise, }\end{cases}
$$

for $\gamma \in N_{1}$ and $t \geqslant T$, is continuous.

Proof. 1 st case. $\gamma \cdot[t, 3 t] \not \subset \operatorname{cl}\left(\bar{N}_{1} \backslash \bar{N}_{0}\right)$. In this case $\gamma \cdot t^{*} \notin \operatorname{cl}\left(\bar{N}_{1} \backslash \bar{N}_{0}\right)$ for some $t^{*}$ with $t<t^{*}<3 t$. Hence there exists a neighborhood $U$ of $\gamma \cdot t^{*}$ in $\Gamma$ such that $U \cap \operatorname{cl}\left(\bar{N}_{1} \backslash \bar{N}_{0}\right)=\varnothing$. By the continuity of the flow, this implies the existence of a neighborhood $W$ of $(\gamma, t)$ in $\Gamma \times[T, \infty)$ such that, whenever $\left(\gamma^{\prime}, t^{\prime}\right) \in W$, then $\gamma^{\prime} \cdot t^{*} \in U$ and $t^{\prime}<t^{*}<3 t^{\prime}$. We conclude that $\gamma^{\prime} \cdot\left[t^{\prime}, 3 t^{\prime}\right] \not \subset \bar{N}_{1} \backslash \bar{N}_{0}$ and hence $f\left(\left[\gamma^{\prime}\right], t^{\prime}\right)=\left[\bar{N}_{0}\right]$ for every $\left(\gamma^{\prime}, t^{\prime}\right) \in W$ with $\gamma^{\prime} \in N_{1}$.

Note that the case $\gamma \cdot[0,2 t] \not \subset \operatorname{cl}\left(N_{1} \backslash N_{0}\right)$ can be treated in a strictly analogous manner. Hence we can assume from now on that

$$
\gamma \cdot[0,2 t] \subset \operatorname{cl}\left(N_{1} \backslash N_{0}\right), \quad \gamma \cdot[t, 3 t] \subset \operatorname{cl}\left(\bar{N}_{1} \backslash \bar{N}_{0}\right) .
$$

2nd case. $\gamma \cdot[t, 3 t] \cap \bar{N}_{0}=\varnothing$. In this case it follows from (4.4) that $\gamma \cdot[t, 3 t] \subset$ $\bar{N}_{1} \backslash \bar{N}_{0}$. By (4.2), this implies $\gamma \cdot 2 t \in N_{1} \backslash N_{0}$ and hence $\gamma \cdot[0,2 t] \subset N_{1} \backslash N_{0}$. Therefore $f([\gamma], t)=\gamma \cdot 3 t \in \bar{N}_{1} \backslash \bar{N}_{0}$. 
Now let $U$ be a neighborhood of $\gamma \cdot 3 t$ in $\Gamma$. Then, by the continuity of the flow, there exists a neighborhood $W$ of $(\gamma, t)$ in $\Gamma \times[T, \infty)$ such that, whenever $\left(\gamma^{\prime}, t^{\prime}\right) \in W$, then

$$
\gamma^{\prime} \cdot\left[0,2 t^{\prime}\right] \cap N_{0}=\varnothing, \quad \gamma^{\prime} \cdot\left[t^{\prime}, 3 t^{\prime}\right] \cap \bar{N}_{0}=\varnothing, \quad \gamma^{\prime} \cdot 3 t^{\prime} \in U .
$$

If $\gamma^{\prime} \in N_{1}$, then we obtain $\gamma^{\prime} \cdot\left[0,2 t^{\prime}\right] \subset N_{1} \backslash N_{0}$ which, by (4.1), implies $\gamma^{\prime} \cdot t^{\prime} \in$ $\bar{N}_{1} \backslash \bar{N}_{0}$ and hence $\gamma^{\prime} \cdot\left[t^{\prime}, 3 t^{\prime}\right] \subset \bar{N}_{1} \backslash \bar{N}_{0}$. Therefore $f\left(\left[\gamma^{\prime}\right], t^{\prime}\right)=\left[\gamma^{\prime} \cdot 3 t^{\prime}\right]=\gamma^{\prime} \cdot 3 t^{\prime}$ $\in U$ for every $\left(\gamma^{\prime}, t^{\prime}\right) \in W$ with $\gamma^{\prime} \in N_{1}$.

3rd case. $\gamma \cdot[t, 3 t] \cap \bar{N}_{0} \neq \varnothing$. In this case it follows from (4.4) that $\gamma \cdot 3 t \in \bar{N}_{0}$. Now let $[U]$ be any neighborhood of $f([\gamma], t)=\left[\bar{N}_{0}\right]$ in $\bar{N}_{1} / \bar{N}_{0}$ and define

$$
U=\left([U] \cap \bar{N}_{1} \backslash \bar{N}_{0}\right) \cup\left(\Gamma \backslash \bar{N}_{1}\right) \cup \bar{N}_{0} .
$$

Then $U$ is a neighborhood of $\bar{N}_{0}$ in $\Gamma$ and

$$
[U]=\left(U \cap \bar{N}_{1} \backslash \bar{N}_{0}\right) \cup\left[\bar{N}_{0}\right] \text {. }
$$

By the continuity of the flow, there exists a neighborhood $W$ of $(\gamma, t)$ in $\Gamma \times[T, \infty)$ such that, whenever $\left(\gamma^{\prime}, t^{\prime}\right) \in W$, then $\gamma^{\prime} \cdot 3 t^{\prime} \in U$. This implies that

$$
f\left(\left[\gamma^{\prime}\right], t^{\prime}\right) \in\left\{\left[\gamma^{\prime} \cdot 3 t^{\prime}\right],\left[\bar{N}_{0}\right]\right\} \subset\left(U \cap \bar{N}_{1} \backslash \bar{N}_{0}\right) \cup\left[\bar{N}_{0}\right]=[U]
$$

for every $\left(\gamma^{\prime}, t^{\prime}\right) \in W$ with $\gamma^{\prime} \in N_{1}$.

Lemma 4.8. Let $\left(N_{1}, N_{0}\right),\left(\bar{N}_{1}, \bar{N}_{0}\right),\left(\overline{\bar{N}}_{1}, \overline{\bar{N}}_{0}\right)$ be index pairs for $S$. Choose $T \geqslant 0$ such that (4.1) and (4.2) are satisfied for $t \geqslant T$ and suppose that the implications

$$
\begin{aligned}
& \gamma \cdot[-t, t] \subset \bar{N}_{1} \backslash \bar{N}_{0} \Rightarrow \gamma \in \overline{\bar{N}}_{1} \backslash \overline{\bar{N}}_{0}, \\
& \gamma \cdot[-t, t] \subset \overline{\bar{N}}_{1} \backslash \overline{\bar{N}}_{0} \Rightarrow \gamma \in \bar{N}_{1} \backslash \bar{N}_{0}
\end{aligned}
$$

hold for $t \geqslant \bar{T}$. Finally, let $f: N_{1} / N_{0} \times[T, \infty) \rightarrow \bar{N}_{1} / \bar{N}_{0}$ be defined by (4.3) and $\bar{f}: \bar{N}_{1} / \bar{N}_{0} \times[\bar{T}, \infty) \rightarrow \overline{\bar{N}}_{1} \backslash \overline{\bar{N}}_{0}$ analogously. Then the following equation holds for $t \geqslant \max \{T, \bar{T}\}$ :

$$
\bar{f}(f([\gamma], t), t)= \begin{cases}{[\gamma \cdot 6 t],} & \gamma \cdot[0,4 t] \subset N_{1} \backslash N_{0}, \gamma \cdot[2 t, 6 t] \subset \overline{\bar{N}}_{1} \backslash \overline{\bar{N}}_{0}, \\ {\left[\overline{\bar{N}}_{0}\right],} & \text { otherwise, } \gamma \in N_{1} .\end{cases}
$$

Proof. We have to show that

$$
\gamma \cdot[0,2 t] \subset N_{1} \backslash N_{0}, \quad \gamma \cdot[t, 5 t] \subset \bar{N}_{1} \backslash \bar{N}_{0}, \quad \gamma \cdot[4 t, 6 t] \subset \overline{\bar{N}}_{1} \backslash \overline{\bar{N}}_{0}
$$

is equivalent to

$$
\gamma \cdot[0,4 t] \subset N_{1} \backslash N_{0}, \quad \gamma \cdot[2 t, 6 t] \subset \overline{\bar{N}}_{1} \backslash \overline{\bar{N}}_{0} .
$$

But this follows immediately from (4.1), (4.2) and (4.5), (4.6).

Now we are in the position to define the index of an isolated invariant set.

DEFINITION 4.9 (INDEX). Let $X \subset \Gamma$ be a metric local flow and let $S$ be an isolated invariant set in $X$. Then the homotopy type $h(S)=\left[N_{1} / N_{0}\right]$ of the pointed space $N_{1} / N_{0},\left(N_{1}, N_{0}\right)$ being an index pair for $S$ in $X$, is said to be the homotopy index of $S$ in $X$. 
The Conley index of $S$ in $X$ is the pair $I(S)=I(S, X)=\left(I_{0}, I_{m}\right)$, where

$$
\begin{array}{r}
I_{0}=\left\{N_{1} / N_{0} \mid\left(N_{1}, N_{0}\right) \text { is an index pair for } S \text { in } X\right\}, \\
I_{m}=\left\{\left[f^{t}\right] \mid N_{1} / N_{0}, \bar{N}_{1} / \bar{N}_{0} \in I_{0} \text { and } f^{t}: N_{1} / N_{0} \rightarrow \bar{N}_{1} / \bar{N}_{0}\right.
\end{array}
$$

is the map defined in Lemma 4.7$\}$.

THEOREM 4.10. Let $X \subset \Gamma$ be a metric local flow and let $S$ be an isolated invariant set in $X$. Then $h(S)$ is independent of the choice of the index pair and $I(S, X)$ is a connected simple system.

Proof. The existence of a homotopy class of maps in $I_{m}$ between any two spaces $N_{1} / N_{0}, \bar{N}_{1} / \bar{N}_{0}$ in $I_{0}$ follows from Lemma 4.7. Lemma 4.8 shows that the composition of any two morphisms in $I_{m}$ is still in $I_{m}$. Finally, it follows from Lemma 4.7 with $N_{1}=\bar{N}_{1}, N_{0}=\bar{N}_{0}, T=0$ that $\left[1_{N_{1} / N_{0}}\right] \in I_{m}$ for every $N_{1} / N_{0} \in I_{0}$. This shows that $I(S, X)$ is a connected simple system. Therefore the morphisms in $I_{m}$ are homotopy equivalences and hence $h(S)$ in independent of the choice of the index pair.

Note that the previous theorem summarizes the paper [6] of Kurland and one of the main results in Conley and Zehnder [4, Theorem 3.2].

The Conley index is a very powerful and elegant tool for studying the structure of isolated invariant sets. A first conclusion is formulated in the corollary below.

Corollary 4.11. Let $X \subset \Gamma$ be a metric local flow and let $S \subset X$ be an isolated invariant set such that $h(S) \neq 0$ (the homotopy class of the one point pointed space). Then $S \neq \varnothing$.

Proof. If $\left(N_{1}, N_{0}\right)$ is an index pair for $S=\varnothing$, then for every $\gamma \in N_{1}$ there exists a $t>0$ such that $\gamma \cdot[0, t] \not \subset \operatorname{cl}\left(N_{1} \backslash N_{0}\right)$. Since $N_{1}$ is compact, this implies that there exists a $T>0$ with $\gamma \cdot[0, T] \cap N_{0} \neq \varnothing$ for all $\gamma \in N_{1}$. Hence the map $f^{t}: N_{1} / N_{0} \rightarrow$ $N_{1} / N_{0}$ given by

$$
f^{t}([\gamma])= \begin{cases}{[\gamma \cdot t],} & \text { if } \gamma \cdot[0, t] \subset N_{1} \backslash N_{0}, \\ {\left[N_{0}\right],} & \text { otherwise, }\end{cases}
$$

for $\gamma \in N_{1}$ and $t \in[0, T]$, defines a homotopy between the zero map $f^{T}$ and the identity $f^{0}$ (Lemma 4.7). This shows that $h(S)=\underline{0}$.

5. A coexact sequence for attractor-repeller pairs. The purpose of this section is to establish a coexact sequence for the Conley index of an attractor-repeller pair along the lines of Theorem 2.5. The first step in this direction is to show that any given index pair can be modified in such a way that it becomes an NDR-pair (\$5.1). Secondly, we have to show that all the maps between index pairs which are involved in the coexact sequence induce morphisms between the corresponding connected simple systems which are independent of the choice of the particular filtration of index pairs $(\$ 5.2)$. 


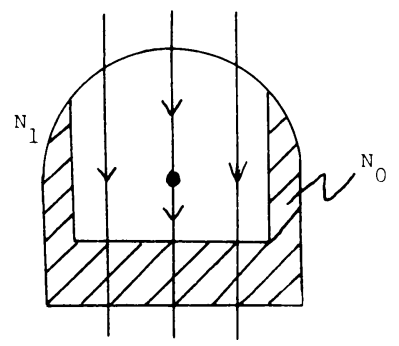

Figure 3

5.1. Regularization of index pairs. We will show that an index pair is an NDR-pair if it is regular in the following sense.

Definition 5.1 (REgUlAR INDEX PAIR). An index pair $\left(N_{1}, N_{0}\right)$ for an isolated invariant set $S$ in a local flow $X \subset \Gamma$ is said to be regular if the function $\tau: N_{1} \rightarrow[0, \infty]$ defined by

$$
\tau(\gamma)= \begin{cases}\sup \left\{t>0 \mid \gamma \cdot[0, t] \subset N_{1} \backslash N_{0}\right\}, & \gamma \in N_{1} \backslash N_{0}, \\ 0, & \gamma \in N_{0},\end{cases}
$$

is continuous.

Figure 3 illustrates an index pair which is not regular.

The next lemma gives a sufficient condition for an index pair to be regular. We were not able to prove that this condition is also necessary and leave this as a conjecture.

Lemma 5.2. Let $\left(N_{1}, N_{0}\right)$ be an index pair for the isolated invariant set $S$ in the local flow $X \subset \Gamma$ and suppose that

$$
\gamma \cdot[0, \varepsilon] \not \subset \operatorname{cl}\left(N_{1} \backslash N_{0}\right) \quad \forall \gamma \in N_{0}, \forall \varepsilon>0 .
$$

Then the index pair $\left(N_{1}, N_{0}\right)$ is regular.

Proof. Let $\tau: N_{1} \rightarrow[0, \infty]$ be defined by (5.1) and let $\gamma \in N_{1}$ be given.

First assume that $0<\tau(\gamma) \leqslant \infty$ and choose $T \in(0, \tau(\gamma))$. Then $\gamma \cdot[0, T] \subset$ $N_{1} \backslash N_{0}$ and thus there exists a neighborhood $U$ of $\gamma$ in $\Gamma$ such that $U \cdot[0, T] \cap N_{0}$ $=\varnothing$. Hence $\gamma^{\prime} \cdot[0, T] \subset N_{1} \backslash N_{0}$ for all $\gamma^{\prime} \in U \cap N_{1}$ and therefore $\tau\left(\gamma^{\prime}\right)>T$ for all $\gamma^{\prime} \in U \cap N_{1}$.

Secondly, suppose that $0 \leqslant \tau(\gamma)<\infty$ and choose $T \in(\tau(\gamma), \infty)$. Then it follows from (5.2) that $\gamma \cdot t \notin \operatorname{cl}\left(N_{1} \backslash N_{0}\right)$ for some $t \in[\tau(\gamma), T]$. This implies that there exists a neighborhood $U$ of $\gamma$ in $\Gamma$ with $U \cdot t \cap \operatorname{cl}\left(N_{1} \backslash N_{0}\right)=\varnothing$. We conclude that $\tau\left(\gamma^{\prime}\right) \leqslant t \leqslant T$ for all $\gamma^{\prime} \in U \cap N_{1}$. This proves the continuity of $\tau$.

In order to transform a given index pair into a regular one we prove the existence of a Lyapunov function (compare Conley [3, p. 33]).

Lemma 5.3. Let $\left(N_{1}, N_{0}\right)$ be an index pair for the isolated invariant set $S$ in the metric local flow $X \subset \Gamma$. Then there exists a continuous (Lyapunov) function $g: N_{1} \rightarrow[0,1]$ such that 


$$
\begin{gathered}
g(\gamma)=1 \Leftrightarrow \gamma \cdot[0, \infty) \subset N_{1} \text { and } \omega(\gamma) \subset S, \\
g(\gamma)=0 \Leftrightarrow \gamma \in N_{0}, \\
t>0, \quad 0<g(\gamma)<1, \quad \gamma \cdot[0, t] \subset N_{1} \Rightarrow g(\gamma \cdot t)<g(\gamma) .
\end{gathered}
$$

Proof. Following the lines of Conley [3, p. 33] we construct the Lyapunov function $g: N_{1} \rightarrow[0,1]$ in three steps.

Step 1 . The function $l: N_{1} \rightarrow[0,1]$ defined by

$$
l(\gamma)=\frac{d\left(\gamma, N_{0}\right)}{d\left(\gamma, N_{0}\right)+d(\gamma, S)}, \quad \gamma \in N_{1},
$$

is continuous and satisfies $N_{0}=l^{-1}(0)$ and $S=l^{-1}(1)$.

Step 2 . The function $k: N_{1} \rightarrow[0,1]$ defined by

$$
k(\gamma)=\sup \left\{l(\gamma \cdot t) \mid t \geqslant 0, \gamma \cdot[0, t] \subset N_{1}\right\}
$$

is continuous and satisfies

$$
\begin{gathered}
k(\gamma)=1 \Leftrightarrow \gamma \cdot[0, \infty) \subset N_{1}, \omega(\gamma) \subset S, \\
k(\gamma)=0 \Leftrightarrow \gamma \in N_{0}, \\
\gamma \cdot[0, t] \subset N_{1} \Rightarrow k(\gamma \cdot t) \leqslant k(\gamma) .
\end{gathered}
$$

Proof. The only nontrivial property of $k$ is the continuity.

Let $\gamma \in N_{1}$ be given, assume first that $0<k(\gamma) \leqslant 1$ and choose $\varepsilon \in(0, k(\gamma))$. Then there exists a $t>0$ such that $\gamma \cdot[0, t] \subset N_{1}$ and $l(\gamma \cdot t)>k(\gamma)-\varepsilon$. Then, of course, $\gamma \cdot[0, t] \subset N_{1} \backslash N_{0}$ and hence there exists a neighborhood $U$ of $\gamma$ in $\Gamma$ such that $U \cdot[0, t] \cap N_{0}=\varnothing$ and $l\left(\gamma^{\prime} \cdot t\right)>k(\gamma)-\varepsilon$ for all $\gamma^{\prime} \in U \cap N_{1}$. This implies $k\left(\gamma^{\prime}\right)>k(\gamma)-\varepsilon$ for all $\gamma^{\prime} \in U \cap N_{1}$.

Secondly, suppose that $0 \leqslant k(\gamma)<1$ and choose $\varepsilon \in(0,1-k(\gamma))$. Furthermore, assume that there is a sequence $\gamma_{n} \in N_{1}$ such that $\gamma=\lim _{n \rightarrow \infty} \gamma_{n}$ and $k\left(\gamma_{n}\right)>k(\gamma)$ $+\varepsilon$ for all $n \in \mathbf{N}$. Then there is a sequence $t_{n} \geqslant 0$ such that $\gamma_{n} \cdot\left[0, t_{n}\right] \subset N_{1} \backslash N_{0}$ and $l\left(\gamma_{n} \cdot t_{n}\right)>k(\gamma)+\varepsilon$. The sequence $t_{n}$ has to be bounded since otherwise $\gamma \cdot[0, \infty) \subset N_{1} \backslash N_{0}$, contradicting $k(\gamma)<1$. Hence the sequence $t_{n}$ has a limit point $t$ and we obtain $\gamma \cdot[0, t] \subset \operatorname{cl}\left(N_{1} \backslash N_{0}\right)$ and $l(\gamma \cdot t) \geqslant k(\gamma)+\varepsilon$, again a contradiction. We conclude that there exists a neighborhood $U$ of $\gamma$ in $\Gamma$ such that $k\left(\gamma^{\prime}\right) \leqslant k(\gamma)+\varepsilon$ for all $\gamma \in U \cap N_{1}$. This proves the continuity of $k$.

Step 3. The function $g: N_{1} \rightarrow[0,1]$ defined by

$$
g(\gamma)=\int_{0}^{t(\gamma)} e^{-\xi} k(\gamma \cdot \xi) d \xi, \quad t(\gamma)=\sup \left\{t>0 \mid \gamma \cdot[0, t] \subset N_{1}\right\},
$$

satisfies the requirements of the lemma.

Proof. The conditions (5.3) and (5.4) are obviously satisfied. In order to establish (5.5), let $\gamma \in N_{1}$ satisfy $0<g(\gamma)<1$ and suppose that

$$
0<t \leqslant t^{*}=\sup \left\{\xi>0 \mid \gamma \cdot[0, \xi] \subset N_{1} \backslash N_{0}\right\}<\infty .
$$

Then

$$
\begin{aligned}
g(\gamma \cdot t) & =\int_{0}^{t^{*}-t} e^{-\xi} k(\gamma \cdot(t+\xi)) d \xi \leqslant \int_{0}^{t^{*}-t} e^{-\xi} k(\gamma \cdot \xi) d \xi \\
& <\int_{0}^{t^{*}} e^{-\xi} k(\gamma \cdot \xi) d \xi=g(\gamma) .
\end{aligned}
$$


Now we prove the continuity of $g$ at $\gamma \in N_{1}$. If $\gamma \in N_{0}$ this follows from the fact that $g\left(\gamma^{\prime}\right) \leqslant k\left(\gamma^{\prime}\right)$ for all $\gamma^{\prime} \in N_{1}$. If $\gamma \cdot[0, \infty) \subset N_{1} \backslash N_{0}$, then the continuity is a consequence of the inequality

$$
\left|g\left(\gamma^{\prime}\right)-g(\gamma)\right| \leqslant \int_{0}^{T}\left|k\left(\gamma^{\prime} \cdot \xi\right)-k(\gamma \cdot \xi)\right| d \xi+2 \int_{T}^{\infty} e^{-\xi} d \xi
$$

for $T$ large and $\gamma^{\prime} \cdot[0, T] \subset N_{1} \backslash N_{0}$. If $\gamma \in N_{1} \backslash N_{0}$ and $\gamma \cdot[0, \infty) \cap N_{0} \neq \varnothing$, then for every $\varepsilon>0$ there exists a $T>0$ such that $\gamma \cdot[0, T] \subset N_{1} \backslash N_{0}$ and $k(\gamma \cdot T)<\varepsilon$. Hence the following inequality holds for all $\gamma^{\prime} \in N_{1}$ with $\gamma^{\prime} \cdot[0, T] \subset N_{1} \backslash N_{0}$ and $k\left(\gamma^{\prime} \cdot T\right)<2 \varepsilon$ :

$$
\left|g\left(\gamma^{\prime}\right)-g(\gamma)\right| \leqslant \int_{0}^{T}\left|k\left(\gamma^{\prime} \cdot \xi\right)-k(\gamma \cdot \xi)\right| d \xi+3 \varepsilon
$$

Thus the continuity of $g$ follows from that of $k$. This completes the proof of Lemma 5.3 .

REMARK 5.4. Let $\left(N_{1}, N_{0}\right)$ be an index pair for the isolated invariant set $S$ in the metric local flow $X \subset \Gamma$ and let $g: N_{1} \rightarrow[0,1]$ be the Lyapunov function of Lemma 5.3. Then we can replace $N_{0}$ by $N_{\varepsilon}=\left\{\gamma \in N_{1} \mid g(\gamma) \leqslant \varepsilon\right\}$ and it follows from Lemma 5.2 that $\left(N_{1}, N_{\varepsilon}\right)$ is a regular index pair for $S$ in $X$ in the sense of Definition 5.1.

5.2. A coexact sequence. Let $S$ be an isolated invariant set in the metric local flow $X \subset \Gamma$, let $A$ be an attractor in $S$ and let $A^{*}$ be the complementary repeller. Then it follows from Corollary 4.4 that there exists a filtration $N_{0} \subset N_{1} \subset N_{2}$ of compact sets in $X$ such that $\left(N_{2}, N_{0}\right)$ is an index pair for $S,\left(N_{1}, N_{0}\right)$ is an index pair for $A$ and $\left(N_{2}, N_{1}\right)$ is an index pair for $A^{*}$. By Remark 5.4, we can assume without loss of generality that the index pair $\left(N_{2}, N_{1}\right)$ is regular. Hence the function $\tau: N_{2} \rightarrow[0, \infty)$ defined by

$$
\tau(\gamma)= \begin{cases}\sup \left\{t>0 \mid \gamma \cdot[0, t] \subset N_{2} \backslash N_{1}\right\}, & \gamma \in N_{2} \backslash N_{1}, \\ 0, & \gamma \in N_{1},\end{cases}
$$

for $\gamma \in N_{2}$, is continuous and the pair $N_{2} / N_{0}, N_{1} / N_{0}$ of pointed spaces with the natural inclusion $\iota$ : $N_{1} / N_{0} \rightarrow N_{2} / N_{0}$ is an NDR-pair. In fact, the functions $r: N_{2} / N_{0} \times[0,1] \rightarrow N_{2} N_{0}$ and $\alpha: N_{2} / N_{0} \rightarrow[0,1]$ defined by

$$
\begin{gathered}
r([\gamma], \sigma)=\left\{\begin{array}{lr}
{[\gamma \cdot \sigma \tau(\gamma)],} & 0 \leqslant \tau(\gamma) \leqslant c, \\
{[\gamma \cdot \sigma c],} & c \leqslant \tau(\gamma) \leqslant \infty,
\end{array}\right. \\
\alpha([\gamma])= \begin{cases}\tau(\gamma) / c, & 0 \leqslant \tau(\gamma) \leqslant c, \\
1, & c \leqslant \tau(\gamma) \leqslant \infty\end{cases}
\end{gathered}
$$

for $\gamma \in N_{2}$ and $\sigma \in[0,1]$, satisfy the requirements of Definition 2.3 (the constant $c>0$ is merely a scaling factor). With these functions the connection map $\delta: N_{2} / N_{1} \rightarrow \sum N_{1} / N_{0}$ defined by equation (2.5) takes the form

$$
\delta([\gamma])= \begin{cases}{[\gamma \cdot \tau(\gamma), 1-\tau(\gamma)],} & 0 \leqslant \tau(\gamma) \leqslant 1, \\ {\left[N_{0} \times 0\right],} & 1 \leqslant \tau(\gamma) \leqslant \infty\end{cases}
$$

for $\gamma \in N_{2}$ (we have chosen $c=2$ ). Hence Theorem 2.5 yields the following result. 
COROllaRy 5.5. Let $A, A^{*}$ be an attractor-repeller pair for the isolated invariant set $S$ in the metric local flow $X \subset \Gamma$. Furthermore, let $N_{0} \subset N_{1} \subset N_{2}$ be a filtration of compact sets in $X$ such that $\left(N_{2}, N_{0}\right)$ and $\left(N_{1}, N_{0}\right)$ are index pairs for $S$ and $A$, respectively, and $\left(N_{2}, N_{1}\right)$ is a regular index pair for $A^{*}$. Finally, let $\iota: N_{1} / N_{0} \rightarrow N_{2} / N_{0}$ and $\pi: N_{2} / N_{0} \rightarrow N_{2} / N_{1}$ be the natural maps and let $\delta: N_{2} / N_{1} \rightarrow \Sigma N_{1} / N_{0}$ be defined by (5.9) and (5.6). Then the following sequence is coexact:

$$
N_{1} / N_{0} \stackrel{\iota}{\rightarrow} N_{2} / N_{0} \stackrel{\pi}{\rightarrow} N_{2} / N_{1} \stackrel{\delta}{\rightarrow} \Sigma N_{1} / N_{0} \stackrel{\Sigma \iota}{\rightarrow} \Sigma N_{2} / N_{0} \stackrel{\Sigma \pi}{\rightarrow} \cdots
$$

REMARK 5.6. Given an index pair $\left(N_{1}, N_{0}\right)$ for the isolated invariant set $S$ in a local flow $X \subset \Gamma$, the pointed space $\Sigma N_{1} / N_{0}$ can be identified with the space $\tilde{N}_{1} / \tilde{N}_{0}$, where

$$
\tilde{N}_{1}=N_{1} \times[-1,1], \quad \tilde{N}_{0}=N_{0} \times[-1,1] \cup N_{1} \times\{-1,1\} .
$$

These two spaces define an index pair for the isolated invariant set $\tilde{S}=S \times 0$ in the local flow $\tilde{X}=X \times \mathbf{R} \subset \tilde{\Gamma}=\Gamma \times \mathbf{R}$, where the flow on $\tilde{\Gamma}$ is defined by $(\gamma, \xi) \cdot t=$ $\left(\gamma \cdot t, \xi e^{t}\right)$ for $\gamma \in \Gamma$ and $\xi, t \in \mathbf{R}$.

Note that each of the maps in the sequence (5.10) induces a morphism between the corresponding connected simple systems. We denote these morphisms still by $\iota: I(A) \rightarrow I(S), \pi: I(S) \rightarrow I\left(A^{*}\right)$ and $\delta: I\left(A^{*}\right) \rightarrow \Sigma I(A)$, respectively. Hence we get the following coexact sequence of connected simple systems:

$$
I(A) \stackrel{\iota}{\rightarrow} I(S) \stackrel{\pi}{\rightarrow} I\left(A^{*}\right) \stackrel{\delta}{\rightarrow} \Sigma I(A) \stackrel{\Sigma \iota}{\rightarrow} \Sigma I(S) \stackrel{\Sigma \pi}{\rightarrow} \cdots .
$$

The whole point in this section is that this sequence is independent of the choice of the particular index filtration. More precisely, we prove the following theorem (compare Kurland [7]).

THEOREM 5.7. Let $A, A^{*}$ be an attractor-repeller for the isolated invariant set $S$ in the metric local flow $X \subset \Gamma$. Furthermore, let $N_{0} \subset N_{1} \subset N_{2}$ be a filtration of compact sets in $X$ such that $\left(N_{2}, N_{0}\right)$ and $\left(N_{1}, N_{0}\right)$ are index pairs for $S$ and $A$, respectively, and $\left(N_{2}, N_{1}\right)$ is a regular index pair for $A^{*}$. Then

(i) the injection $\iota: N_{1} / N_{0} \rightarrow N_{2} / N_{0}$ induces a morphism between $I(A, X)$ and $I(S, X)$ which is independent of the choice of the filtration,

(ii) the projection $\pi: N_{2} / N_{0} \rightarrow N_{2} / N_{1}$ induces a morphism between $I(S, X)$ and $I\left(A^{*}, X\right)$ which is independent of the choice of the filtration, and

(iii) the connection map $\delta: N_{2} / N_{1} \rightarrow \Sigma N_{1} / N_{0}$ defined by (5.9) and (5.6) induces a morphism between $I\left(A^{*}, X\right)$ and $\Sigma I(A, X)$ which is independent of the choice of the filtration.

Proof. Let $\bar{N}_{0} \subset \bar{N}_{1} \subset \bar{N}_{2}$ be another filtration of compact sets in $X$ such that $\left(\bar{N}_{2}, \bar{N}_{0}\right)$ and $\left(\bar{N}_{1}, \bar{N}_{0}\right)$ are index pairs for $S$ and $A$, respectively, and $\left(\bar{N}_{2}, \bar{N}_{1}\right)$ is a regular index pair for $A^{*}$. Furthermore, let $i: \bar{N}_{1} / \bar{N}_{0} \rightarrow \bar{N}_{2} / \bar{N}_{0}, \bar{\pi}: \bar{N}_{2} / \bar{N}_{0} \rightarrow \bar{N}_{2} / \bar{N}_{1}$, $\bar{\delta}: \bar{N}_{2} / \bar{N}_{1} \rightarrow \Sigma \bar{N}_{1} / \bar{N}_{0}$ denote the associated maps and let $\bar{\tau}: \bar{N}_{2} \rightarrow[0, \infty]$ denote the "entrance-time" for the subset $\bar{N}_{1}$, defined analogously to (5.6). 
In order to prove statement (i), choose $T>0$ such that the implications

$$
\begin{aligned}
& \gamma \cdot[-T, T] \subset N_{1} \backslash N_{0} \Rightarrow \gamma \in \bar{N}_{1} \backslash \bar{N}_{0}, \\
& \gamma \cdot[-T, T] \subset \bar{N}_{1} \backslash \bar{N}_{0} \Rightarrow \gamma \in N_{1} \backslash N_{0}, \\
& \gamma \cdot[-T, T] \subset N_{2} \backslash N_{0} \Rightarrow \gamma \in \bar{N}_{2} \backslash \bar{N}_{0}, \\
& \gamma \cdot[-T, T] \subset \bar{N}_{2} \backslash \bar{N}_{0} \Rightarrow \gamma \in N_{2} \backslash N_{0}
\end{aligned}
$$

are satisfied and let $f^{t}: N_{1} / N_{0} \rightarrow \bar{N}_{1} / \bar{N}_{0}$ and $g^{t}: \bar{N}_{2} / \bar{N}_{0} \rightarrow N_{2} / N_{0}$ be defined by

$$
\begin{aligned}
& f^{t}([\gamma])= \begin{cases}{[\gamma \cdot 3 t],} & \gamma \cdot[0,2 t] \subset N_{1} \backslash N_{0}, \gamma \cdot[t, 3 t] \subset \bar{N}_{1} \backslash \bar{N}_{0}, \\
{\left[\bar{N}_{0}\right],} & \text { otherwise }, \gamma \in N_{1},\end{cases} \\
& g^{t}([\gamma])= \begin{cases}{[\gamma \cdot 3 t],} & \gamma \cdot[0,2 t] \subset \bar{N}_{2} \backslash \bar{N}_{0}, \gamma \cdot[t, 3 t] \subset N_{2} \backslash N_{0}, \\
{\left[N_{0}\right],} & \text { otherwise, } \gamma \in \bar{N}_{2},\end{cases}
\end{aligned}
$$

both for $t \geqslant T$. Then we have to show that $g^{t} \circ \imath \circ f^{t}: N_{1} / N_{0} \rightarrow N_{2} / N_{0}$ is homotopic to the canonical injection $\iota: N_{1} / N_{0} \rightarrow N_{2} / N_{0}$. In fact, it follows from (5.12)-(5.15) that for every $\gamma \in N_{1}$ and every $t \geqslant T$

$$
\begin{array}{ll}
\gamma \cdot[0,2 t] \subset N_{1} \backslash N_{0}, & \gamma \cdot[t, 3 t] \subset \bar{N}_{1} \backslash \bar{N}_{0}, \\
\gamma \cdot[3 t, 5 t] \subset \bar{N}_{2} \backslash \bar{N}_{0}, & \gamma \cdot[4 t, 6 t] \subset N_{2} \backslash N_{0}
\end{array}
$$

is equivalent to $\gamma \cdot[0,6 t] \subset N_{2} \backslash N_{0}$. Hence

$$
g^{t} \circ i \circ f^{t}([\gamma])= \begin{cases}{[\gamma \cdot 6 t],} & \gamma \cdot[0,6 t] \subset N_{2} \backslash N_{0}, \\ {\left[N_{0}\right],} & \text { otherwise, } \gamma \in N_{1} .\end{cases}
$$

It follows from Lemma 4.7 that this map is homotopic to $\iota: N_{1} / N_{0} \rightarrow N_{2} / N_{0}$. Thus we have established (i).

In order to prove (ii), suppose that the implications (5.14), (5.15) and

$$
\begin{aligned}
& \gamma \cdot[-T, T] \subset N_{2} \backslash N_{1} \Rightarrow \gamma \in \bar{N}_{2} \backslash \bar{N}_{1}, \\
& \gamma \cdot[-T, T] \subset \bar{N}_{2} \backslash \bar{N}_{1} \Rightarrow \gamma \in N_{2} \backslash N_{1}
\end{aligned}
$$

are satisfied, let $g^{t}: \bar{N}_{2} / \bar{N}_{0} \rightarrow N_{2} / N_{0}$ be defined by (5.17) and $h^{t}: N_{2} / N_{1} \rightarrow \bar{N}_{2} / \bar{N}_{1}$ by

$$
h^{t}([\gamma])= \begin{cases}{[\gamma \cdot 3 t],} & \gamma \cdot[0,2 t] \subset N_{2} \backslash N_{1}, \gamma \cdot[t, 3 t] \subset \bar{N}_{2} \backslash \bar{N}_{1}, \\ {\left[\bar{N}_{1}\right],} & \text { otherwise, } \gamma \in N_{2},\end{cases}
$$

both for $t \geqslant T$. Then we have to show that $h^{t} \circ \pi \circ g^{t}: \bar{N}_{2} / \bar{N}_{0} \rightarrow \bar{N}_{2} / \bar{N}_{1}$ is homotopic to the canonical projection $\bar{\pi}: \bar{N}_{2} / \bar{N}_{0} \rightarrow \bar{N}_{2} / \bar{N}_{1}$. In fact, it follows from (5.14), (5.15) and (5.18), (5.19) that for every $\gamma \in \bar{N}_{2}$ and every $t \geqslant T$

$$
\begin{array}{ll}
\gamma \cdot[0,2 t] \subset \bar{N}_{2} \backslash \bar{N}_{0}, & \gamma \cdot[t, 3 t] \subset N_{2} \backslash N_{0}, \\
\gamma \cdot[3 t, 5 t] \subset N_{2} \backslash N_{1}, & \gamma \cdot[4 t, 6 t] \subset \bar{N}_{2} \backslash \bar{N}_{1}
\end{array}
$$

is equivalent to $\gamma \cdot[0,6 t] \subset \bar{N}_{2} \backslash \bar{N}_{1}$. Hence

$$
h^{t} \circ \pi \circ g^{t}([\gamma])= \begin{cases}{[\gamma \cdot 6 t],} & \gamma \cdot[0,6 t] \subset \bar{N}_{2} \backslash \bar{N}_{1}, \\ {\left[\bar{N}_{1}\right],} & \text { otherwise, } \gamma \in \bar{N}_{2} .\end{cases}
$$


It follows again from Lemma 4.7 that this map is homotopic to $\bar{\pi}: \bar{N}_{2} / \bar{N}_{0} \rightarrow \bar{N}_{2} / \bar{N}_{1}$. This proves statement (ii).

In order to prove statement (iii), choose $T>0$ such that the implications (5.12)-(5.15) as well as (5.18), (5.19) are satisfied, let $h^{t}: N_{2} / N_{1} \rightarrow \bar{N}_{2} / \bar{N}_{1}$ be defined by (5.20) and $f^{t}: \bar{N}_{1} / \bar{N}_{0} \rightarrow N_{1} / N_{0}$ by

$$
f^{\prime}([\gamma])= \begin{cases}{[\gamma \cdot 3 t]} & \gamma \cdot[0,2 t] \subset \bar{N}_{1} \backslash \bar{N}_{0}, \gamma \cdot[t, 3 t] \subset N_{1} \backslash N_{0}, \\ {\left[N_{0}\right],} & \text { otherwise, } \gamma \in \bar{N}_{1},\end{cases}
$$

both for $t \geqslant T$. Then we have to show that $\Sigma f^{t} \circ \bar{\delta} \circ h^{t}: N_{2} / N_{1} \rightarrow \Sigma N_{1} / N_{0}$ is homotopic to $\delta$. First of all, it follows from Lemma 4.7 that the map $\delta=\delta^{0}$ is homotopic to the map $\delta^{t}: N_{2} / N_{1} \rightarrow \Sigma N_{1} / N_{0}$ which is defined by

$$
\delta^{t}([\gamma])=\left\{\begin{array}{c}
{[\gamma \cdot(6 t+\tau(\gamma \cdot 3 t)), 1-\tau(\gamma \cdot 3 t)], \quad \text { if } \gamma \cdot[0,3 t] \subset N_{2} \backslash N_{1},} \\
0<\tau(\gamma \cdot 3 t)<1 \text { and } \gamma \cdot[0,6 t+\tau(\gamma \cdot 3 t)] \subset N_{2} \backslash N_{0}, \\
{\left[N_{0} \times 0\right], \quad \text { otherwise, } \gamma \in N_{2} .}
\end{array}\right.
$$

A homotopy between $\Sigma f^{t} \circ \bar{\delta} \circ h^{t}$ and $\delta^{t}$ is given by the family of maps $F^{\sigma}: N_{2} / N_{1} \rightarrow \Sigma N_{1} / N_{0}, 0 \leqslant \sigma \leqslant 1$, defined by

$$
F^{\sigma}([\gamma])=\left\{\begin{array}{c}
{\left[\gamma \cdot\left(6 t+\tau^{\sigma}(\gamma)\right), 1-\tau^{\sigma}(\gamma)\right], \quad \text { if } \gamma \cdot[0,2 t] \subset N_{2} \backslash N_{1},} \\
0<\tau^{\sigma}(\gamma)<1, \text { and } \gamma \cdot\left[0,6 t+\tau^{\sigma}(\gamma)\right] \subset N_{2} \backslash N_{0}, \\
{\left[N_{0} \times 0\right], \quad \text { otherwise, } \gamma \in N_{2},}
\end{array}\right.
$$

where

$$
\tau^{\sigma}(\gamma)=(1-\sigma) \bar{\tau}(\gamma \cdot t)+\sigma \tau(\gamma \cdot t)-2 t, \quad \gamma \cdot[0,2 t] \subset N_{2} \backslash N_{1} .
$$

Note that $\bar{\tau}(\gamma \cdot t)$ makes sense since $\gamma \cdot[0,2 t] \subset N_{2} \backslash N_{1}$ implies that $\gamma \cdot t \in \bar{N}_{2} \backslash \bar{N}_{1}$. Of course, $F^{1}=\delta^{t}$. Furthermore it follows from (5.13)-(5.15) and (5.18) that for all $\gamma \in N_{2}$ and all $t \geqslant T$

$$
\begin{gathered}
\gamma \cdot[0,2 t] \subset N_{2} \backslash N_{1}, \quad 2 t<\bar{\tau}(\gamma \cdot t)<2 t+1, \\
\gamma \cdot[0,6 t+\bar{\tau}(\gamma \cdot 3 t)] \subset N_{2} \backslash N_{0}
\end{gathered}
$$

is equivalent to

$$
\begin{gathered}
\gamma \cdot[0,2 t] \subset N_{2} \backslash N_{1}, \quad \gamma \cdot[t, 3 t] \subset \bar{N}_{2} \backslash \bar{N}_{1}, \\
0<\bar{\tau}(\gamma \cdot 3 t)<1, \quad \gamma \cdot[3 t+\bar{\tau}(\gamma \cdot 3 t), 5 t+\bar{\tau}(\gamma \cdot 3 t)] \subset \bar{N}_{1} \backslash \bar{N}_{0}, \\
\gamma \cdot[4 t+\bar{\tau}(\gamma \cdot 3 t), 6 t+\bar{\tau}(\gamma \cdot 3 t)] \subset N_{1} \backslash N_{0} .
\end{gathered}
$$

This implies that $F^{0}=\Sigma f^{t} \circ \bar{\delta} \circ h^{t}$. Hence it remains to show that $F^{\sigma}$ is continuous on the domain $N_{2} / N_{1} \times[0,1]$. We prove this for the case $t>2 T$ and $t \geqslant 1$ in seven steps.

Step 1. If $\gamma \cdot[0,2 t] \subset N_{2} \backslash N_{1}$ and $t \geqslant 2 T$, then $|\tau(\gamma \cdot t)-\bar{\tau}(\gamma \cdot t)| \leqslant T$.

PROOF. First, $\gamma \cdot[0, t+\tau(\gamma \cdot t)) \subset N_{2} \backslash N_{1}$ and hence $\gamma \cdot[T, t-T+\tau(\gamma \cdot t))$ $\subset \bar{N}_{2} \backslash \bar{N}_{1}$, by (5.18). This implies $\bar{\tau}(\gamma \cdot t) \geqslant \tau(\gamma \cdot t)-T>T$. Furthermore, $\gamma \cdot[T, t+\bar{\tau}(\gamma \cdot t)) \subset \bar{N}_{2} \backslash \bar{N}_{1}$ and hence $\gamma \cdot[2 T, t-T+\bar{\tau}(\gamma \cdot t)) \subset N_{2} \backslash N_{1}$, by (5.19). This shows that $\tau(\gamma \cdot t) \geqslant \bar{\tau}(\gamma \cdot t)-T$. 
Step 2. If $t>2 T, \gamma \cdot[0,2 t] \subset N_{2} \backslash N_{1}$ and $0<\tau^{\sigma}(\gamma)<1$, then

$$
\begin{aligned}
2 t-(1-\sigma) T<\tau(\gamma \cdot t) & <2 t+(1-\sigma) T+1, \\
2 t-\sigma T<\bar{\tau}(\gamma \cdot t) & <2 t+\sigma T+1 .
\end{aligned}
$$

Proof. Since $\tau(\gamma \cdot t) \leqslant \bar{\tau}(\gamma \cdot t)+T$ (Step 1), we have

$$
\begin{aligned}
0 & <\tau^{\sigma}(\gamma) \leqslant(1-\sigma) \bar{\tau}(\gamma \cdot t)+\sigma(T+\bar{\tau}(\gamma \cdot t))-2 t \\
& =\bar{\tau}(\gamma \cdot t)+\sigma T-2 t
\end{aligned}
$$

and

$$
\begin{aligned}
1 & >\tau^{\sigma}(\gamma) \geqslant(1-\sigma)(\tau(\gamma \cdot t)-T)+\sigma \tau(\gamma \cdot t)-2 t \\
& =\tau(\gamma \cdot t)-(1-\sigma) T-2 t .
\end{aligned}
$$

Since $\tau(\gamma \cdot t) \geqslant \bar{\tau}(\gamma \cdot t)-T$, we get

$$
\begin{aligned}
1 & >\tau^{\sigma}(\gamma) \geqslant(1-\sigma) \bar{\tau}(\gamma \cdot t)+\sigma(\bar{\tau}(\gamma \cdot t)-T)-2 t \\
& =\bar{\tau}(\gamma \cdot t)-2 t-\sigma T
\end{aligned}
$$

and

$$
\begin{aligned}
0 & <\tau^{\sigma}(\gamma) \leqslant(1-\sigma)(\tau(\gamma \cdot t)+T)+\sigma \tau(\gamma \cdot t)-2 t \\
& =\tau(\gamma \cdot t)+(1-\sigma) T-2 t .
\end{aligned}
$$

Step 3. If $\tau(\gamma)<3 t-(1-\sigma) T$, then $F^{\sigma}: N_{2} / N_{1} \rightarrow \Sigma N_{1} / N_{0}$ is continuous at $([\gamma], \sigma)$.

Proof. Choose a neighborhood $W$ of $(\gamma, \sigma)$ in $N_{2} \times[0,1]$ such that, whenever $\left(\gamma^{\prime}, \sigma^{\prime}\right) \in W$, then $\tau\left(\gamma^{\prime}\right)<3 t-\left(1-\sigma^{\prime}\right) T$. Then it follows from Step 2 that either $\gamma^{\prime} \cdot[0,2 t] \not \subset N_{2} \backslash N_{1}$ or $\tau^{\sigma^{\prime}}\left(\gamma^{\prime}\right) \notin(0,1)$. Hence $F^{\sigma^{\prime}}\left(\left[\gamma^{\prime}\right]\right)=\left[N_{0} \times 0\right]$ for all $\left(\gamma^{\prime}, \sigma^{\prime}\right) \in$ $W$.

Step 4. From now on we can assume that $\tau(\gamma) \geqslant 3 t-(1-\sigma) T$ and hence

$$
\gamma \cdot[0,2 t] \subset N_{2} \backslash N_{1} .
$$

If (5.22) is satisfied and $\tau^{\sigma}(\gamma) \notin(0,1)$, then the function $F$ is continuous at $([\gamma], \sigma)$.

Proof. First note that $F^{\sigma}([\gamma])=\left[N_{0} \times 0\right]$. Secondly, note that the function $\tau^{\sigma}(\gamma)$ is continuous on the domain $\left\{(\gamma, \sigma) \in N_{2} \times[0,1] \mid \gamma \cdot[0,2 t] \cap N_{1}=\varnothing\right\}$. Hence for every $\varepsilon>0$, there exists a neighborhood $W$ of $(\gamma, \sigma)$ in $N_{2} \times[0,1]$ such that, whenever $\left(\gamma^{\prime}, \sigma^{\prime}\right) \in W$, then $\gamma^{\prime} \cdot[0,2 t] \cap N_{1}=\varnothing$ and $\tau^{\sigma^{\prime}}\left(\gamma^{\prime}\right) \notin[\varepsilon, 1-\varepsilon]$. This proves Step 4.

Step 5. From now on we can assume that (5.22) is satisfied and

$$
0<\tau^{\sigma}(\gamma)<1 .
$$

If moreover $\gamma \cdot\left[0,6 t+\tau^{\sigma}(\gamma)\right] \not \subset \mathrm{cl}\left(N_{2} \backslash N_{0}\right)$, then $F$ is continuous at $([\gamma], \sigma)$.

Proof. There is a $t^{*}<6 t+\tau^{\sigma}(\gamma)$ such that $\gamma \cdot t^{*} \notin \operatorname{cl}\left(N_{2} \backslash N_{0}\right)$. Hence there is a neighborhood $W$ of $(\gamma, \sigma)$ in $N_{2} \times[0,1]$ such that, whenever $\left(\gamma^{\prime}, \sigma^{\prime}\right) \in W$, then $\gamma^{\prime} \cdot[0,2 t] \subset N_{2} \backslash N_{1}, 0<\tau^{\sigma^{\prime}}\left(\gamma^{\prime}\right)<1, t^{*}<6 t+\tau^{\sigma^{\prime}}\left(\gamma^{\prime}\right)$, and $\gamma^{\prime} \cdot t^{*} \notin \operatorname{cl}\left(N_{2} \backslash N_{0}\right)$. Hence $F^{\sigma^{\prime}}\left(\left[\gamma^{\prime}\right]\right)=\left[N_{0} \times 0\right]$ for all $\left(\gamma^{\prime}, \sigma^{\prime}\right) \in W$.

Step 6. If (5.22) and (5.23) are satisfied and if $\gamma \cdot\left[0,6 t+\tau^{\sigma}(\gamma)\right] \subset N_{2} \backslash N_{0}$, then $F$ is continuous at $([\gamma], \sigma)$. 
Proof. First note that, by Step $2, \tau(\gamma)<3 t+(1-\sigma) T+1<4 t+1$ and therefore $\gamma \cdot\left(6 t+\tau^{\sigma}(\gamma)\right) \in N_{1}$ (recall that $t \geqslant 1$ ). Now let $U$ be a neighborhood of $\gamma \cdot\left(6 t+\tau^{\sigma}(\gamma)\right)$ in $\Gamma$. Moreover, choose a neighborhood $V$ of $\gamma \cdot\left(6 t+\tau^{\sigma}(\gamma)\right)$ in $\Gamma$ and an $\varepsilon>0$ such that

$$
\begin{gathered}
V \cdot[-\varepsilon, \varepsilon] \subset U, \quad \gamma \cdot\left[0,6 t+\tau^{\sigma}(\gamma)+\varepsilon\right] \subset N_{2} \backslash N_{0}, \\
{\left[\tau^{\sigma}(\gamma)-\varepsilon, \tau^{\sigma}(\gamma)+\varepsilon\right] \subset(0,1) .}
\end{gathered}
$$

Then there exists a neighborhood $W$ of $(\gamma, \sigma)$ in $N_{2} \times[0,1]$, such that, whenever $\left(\gamma^{\prime}, \sigma^{\prime}\right) \in W$, then

$$
\begin{gathered}
\gamma^{\prime} \cdot[0,2 t] \subset N_{2} \backslash N_{1}, \quad \gamma^{\prime} \cdot\left[0,6 t+\tau^{\sigma}(\gamma)+\varepsilon\right] \subset N_{2} \backslash N_{0}, \\
\left|\tau^{\sigma^{\prime}}\left(\gamma^{\prime}\right)-\tau^{\sigma}(\gamma)\right| \leqslant \varepsilon, \quad \gamma^{\prime} \cdot\left(6 t+\tau^{\sigma}(\gamma)\right) \in V .
\end{gathered}
$$

Then we get $\gamma^{\prime} \cdot[0,2 t] \subset N_{2} \backslash N_{1}, 0<\tau^{\sigma^{\prime}}\left(\gamma^{\prime}\right)<1, \gamma^{\prime} \cdot\left[0,6 t+\tau^{\sigma^{\prime}}\left(\gamma^{\prime}\right)\right] \subset N_{2} \backslash N_{0}$ and $\gamma^{\prime} \cdot\left(6 t+\tau^{\sigma^{\prime}}\left(\gamma^{\prime}\right)\right) \in U,\left|\tau^{\sigma^{\prime}}\left(\gamma^{\prime}\right)-\tau^{\circ}(\gamma)\right| \leqslant \varepsilon$ for all $\left(\gamma^{\prime}, \sigma^{\prime}\right) \in W$ and hence -

$$
\begin{aligned}
F^{\sigma^{\prime}}\left(\left[\gamma^{\prime}\right]\right) & =\left[\gamma^{\prime} \cdot\left(6 t+\tau^{\sigma^{\prime}}\left(\gamma^{\prime}\right)\right), 1-\tau^{\sigma^{\prime}}\left(\gamma^{\prime}\right)\right] \\
& \in\left(U \cap N_{1}\right) \times\left[1-\tau^{\sigma}(\gamma)-\varepsilon, 1-\tau^{\sigma}(\gamma)+\varepsilon\right] .
\end{aligned}
$$

This proves Step 6.

Step 7. If (5.22) and (5.23) are satisfied and if $\gamma \cdot\left[0,6 t+\tau^{\sigma}(\gamma)\right] \subset \operatorname{cl}\left(N_{2} \backslash N_{0}\right)$, $\gamma \cdot\left(6 t+\tau^{\sigma}(\gamma)\right) \in N_{0}$, then $F$ is continuous at $([\gamma], \sigma)$.

Proof. Note that $F^{\sigma}([\gamma])=\left[N_{0} \times 0\right]$ and choose a neighborhood $[U]$ of $\left[N_{0}\right]$ in $N_{1} / N_{0}$. Then

$$
U=\left([U] \cap N_{1} \backslash N_{0}\right) \cup N_{0} \cup\left(\Gamma \backslash N_{1}\right)
$$

is a neighborhood of $N_{0}$ in $\Gamma$ and satisfies

$$
[U]=\left(U \cap N_{1} \backslash N_{0}\right) \cup\left[N_{0}\right] .
$$

Now choose a neighborhood $V$ of $N_{0}$ in $\Gamma$ and an $\varepsilon>0$ such that $V \cdot[-\varepsilon, \varepsilon] \subset U$. Then there exists a neighborhood $W$ of $(\gamma, \sigma)$ in $N_{2} \times[0,1]$ such that whenever $\left(\gamma^{\prime}, \sigma^{\prime}\right) \in W$, then

$$
\begin{gathered}
\gamma^{\prime} \cdot[0,2 t] \subset N_{2} \backslash N_{1}, \quad 0<\tau^{\sigma^{\prime}}\left(\gamma^{\prime}\right)<1, \\
\left|\tau^{\sigma}(\gamma)-\tau^{\sigma^{\prime}}\left(\gamma^{\prime}\right)\right|<\varepsilon, \quad \gamma^{\prime} \cdot\left(6 t+\tau^{\sigma}(\gamma)\right) \in V .
\end{gathered}
$$

Hence we obtain $\gamma^{\prime} \cdot[0,2 t] \subset N_{2} \backslash N_{1}, 0<\tau^{\sigma^{\prime}}\left(\gamma^{\prime}\right)<1, \gamma^{\prime} \cdot\left(6 t+\tau^{\sigma^{\prime}}\left(\gamma^{\prime}\right)\right) \in U$ and therefore

$$
\begin{aligned}
F^{\sigma^{\prime}}\left(\left[\gamma^{\prime}\right]\right) & \in\left\{\left[\gamma^{\prime} \cdot\left(6 t+\tau^{\sigma^{\prime}}\left(\gamma^{\prime}\right)\right), 1-\tau^{\sigma^{\prime}}\left(\gamma^{\prime}\right)\right],\left[N_{0} \times 0\right]\right\} \\
& \subset\left(U \cap N_{1} \backslash N_{0}\right) \times[0,1] \cup\left[N_{0} \times 0\right] \\
& =[U] \times[0,1]
\end{aligned}
$$

for all $\left(\gamma^{\prime}, \sigma^{\prime}\right) \in W$. This proves Theorem 5.7.

The final result of this section shows an interesting relation between the coexact sequence (5.11) and the structure of the isolated invariant set $S$ (see Conley [3, p. 62]). 
THEOREM 5.8. Let $A, A^{*}$ be an attractor-repeller pair for the isolated invariant set $S$ in the metric local flow $X \subset \Gamma$ and suppose that $S=A \cup A^{*}$. Then $h(S)=$ $h(A) \vee h\left(A^{*}\right)$ and the connection map $\delta$ defined by (5.9) and (5.6) is homotopic to the constant map.

Proof. If $S=A \cup A^{*}$, then there exist index pairs $\left(N_{1}, N_{0}\right)$ and $\left(N_{1}^{*}, N_{0}^{*}\right)$ for $A$ and $A^{*}$, respectively, such that $N_{1} \cap N_{1}^{*}=\varnothing$. In this case $\left(N_{1} \cup N_{1}^{*}, N_{0} \cup N_{0}^{*}\right)$ is an index pair for $S$ and hence

$$
h(S)=\left[N_{1} \cup N_{1}^{*} / N_{0} \cup N_{0}^{*}\right]=\left[N_{1} / N_{0}\right] \vee\left[N_{1}^{*} / N_{0}^{*}\right]=h(A) \vee h\left(A^{*}\right) .
$$

Now let $N_{0} \subset N_{1} \subset N_{2}$ be a filtration of compact sets in $X$ such that $\left(N_{2}, N_{0}\right)$ and $\left(N_{1}, N_{0}\right)$ are index pairs for $S$ and $A$, respectively, and $\left(N_{2}, N_{1}\right)$ is a regular index pair for $A^{*}$. Then there exists a $T>0$ such that

$$
\gamma \cdot[-T, 0] \subset \operatorname{cl}\left(N_{2} \backslash N_{1}\right), \quad \gamma \in N_{1} \Rightarrow \gamma \cdot[0, T] \cap N_{0} \neq \varnothing .
$$

Otherwise, there would exist sequences $\gamma_{n} \in N_{1}, t_{n}>0$, such that $t_{n}$ tends to infinity and $\gamma_{n} \cdot\left[-t_{n}, 0\right] \subset \operatorname{cl}\left(N_{2} \backslash N_{1}\right), \gamma_{n} \cdot\left[0, t_{n}\right] \cap N_{0}=\varnothing$. Any limit point $\gamma$ of $\gamma_{n}$ would then satisfy $\gamma \cdot(-\infty, 0] \subset \operatorname{cl}\left(N_{2} \backslash N_{1}\right), \gamma \cdot[0, \infty) \subset \operatorname{cl}\left(N_{1} \backslash N_{0}\right)$ and therefore $\omega^{*}(\gamma) \subset A^{*}, \omega(\gamma) \subset A, \gamma \cdot \mathbf{R} \subset \operatorname{cl}\left(N_{2} \backslash N_{0}\right)$. But this would imply $\gamma \in S \backslash\left(A \cup A^{*}\right)$, contradicting $S=A \cup A^{*}$.

Now let $\delta: N_{2} / N_{1} \rightarrow \Sigma N_{1} / N_{0}$ be given by (5.9) and (5.6) and let us define the maps $\delta_{t}: N_{2} / N_{1} \rightarrow \sum N_{1} / N_{0}, f_{t}: N_{1} / N_{0} \rightarrow N_{1} / N_{0}$ by

$$
\begin{gathered}
\delta_{t}([\gamma])= \begin{cases}{[\gamma \cdot \tau(\gamma), t+1-\tau(\gamma)],} & t<\tau(\gamma)<t+1, \\
{\left[N_{0} \times 0\right],} & \text { otherwise, }\end{cases} \\
f_{t}([\gamma])= \begin{cases}{[\gamma \cdot t],} & \text { if } \gamma \cdot[0, t] \subset N_{1} \backslash N_{0}, \\
{\left[N_{0}\right],} & \text { otherwise, }\end{cases}
\end{gathered}
$$

for $0 \leqslant t \leqslant T$. Then $\delta_{0}=\delta, f_{0}$ is the identity map, and it follows from (5.24) that $\Sigma f_{T} \circ \delta_{T}$ is the constant map. Hence the continuous family of maps $\Sigma f_{t} \circ \delta_{t}$, $0 \leqslant t \leqslant T$, defines a homotopy between $\delta=\Sigma f_{0} \circ \delta_{0}$ and $c=\Sigma f_{T} \circ \delta_{T}$.

6. Continuation. The aim of this section is to establish the invariance of the Conley index for isolated invariant sets under (small) perturbations of the flow. For this purpose we first collect some elementary properties of parametrized flows and, in particular, make precise what we mean by continuation of isolated invariant sets (compare Conley [3, Chapter IV.1]).

6.1. Parametrized flows. Throughout this section we shall assume that $\Lambda$ is a compact, locally contractible, connected, metric space and $X$ is a locally compact metric space. Furthermore, we assume that $\Gamma$ is a flow and $X \times \Lambda \subset \Gamma$ is a local flow with the property that if $(x, \lambda) \cdot t \in X \times \lambda$, then $(x, \lambda) \cdot t \in X \times \lambda$ for all $x \in X, \lambda \in \Lambda, t \in \mathbf{R}$. Then, of course $X \times \lambda \subset \Gamma$ is a local flow for every $\lambda \in \Lambda$. We will always denote by

$$
\pi_{X}: X \times \Lambda \rightarrow X, \quad \pi_{\Lambda}: X \times \Lambda \rightarrow \Lambda
$$

the canonical projection maps. 


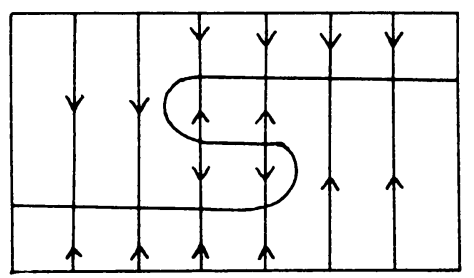

Figure 4

LeMMA 6.1. For any compact set $N \subset X$ the set $\Lambda(N)=\{\lambda \in \Lambda \mid N \times \lambda$ is an isolating neighborhood in $X \times \lambda$ \} is open in $\Lambda$.

Proof. Suppose that there is a $\lambda \in \Lambda$, a sequence $\lambda_{k} \in \Lambda$ converging to $\lambda$ and a compact set $N \subset X$ such that $N \times \lambda$ is an isolating neighborhood in $X \times \lambda$ but $N \times \lambda_{k}$ is not an isolating neighborhood in $X \times \lambda_{k}$. Then there exists a sequence $x_{k} \in N \cap \operatorname{cl}(X \backslash N)$ such that $\left(x_{k}, \lambda_{k}\right) \cdot \mathbf{R} \subset N \times \lambda_{k}$ for all $k \in \mathbf{N}$. Any limit point $x \in N \cap \operatorname{cl}(X \backslash N)$ of $x_{k}$ satisfies $(x, \lambda) \cdot \mathbf{R} \subset N \times \lambda$, contradicting the fact that $N \times \lambda$ is an isolating neighborhood in $X \times \lambda$.

Let us now introduce the set $\mathscr{S}=\{S \times \lambda \mid \lambda \in \Lambda, S \subset X$ compact, $S \times \lambda$ is an isolated invariant set in $X \times \lambda\}$ of isolated invariant sets in $X \times \Lambda$. For every compact set $N \subset X$ let us define the map

$$
\sigma_{N}: \Lambda(N) \rightarrow \mathscr{S}, \quad \sigma_{N}(\lambda)=I(N \times \lambda) .
$$

Then we consider on the space $\mathscr{S}$ the topology which is generated by the sets $\left\{\sigma_{N}(U) \mid N \subset X\right.$ compact, $U \subset \Lambda(N)$ open $\}$. Note that $\mathscr{S}$ is not necessarily Hausdorff as Figure 4 shows.

LeMmA 6.2. Let $N, \bar{N}$ be compact subsets of $X$ and let $U \subset \Lambda(N)$ and $\bar{U} \subset \Lambda(\bar{N})$ be open. Then the following statements hold.

(i) $\Lambda(N, \bar{N})=\{\lambda \in \Lambda(N) \cap \Lambda(\bar{N}) \mid I(N \times \lambda)=I(\bar{N} \times \lambda)\}$ is open in $\Lambda$.

(ii) $\sigma_{N}(U) \cap \sigma_{\bar{N}}(\bar{U})=\sigma_{N}(U \cap \bar{U} \cap \Lambda(N, \bar{N}))$.

(iii) $\sigma_{N}: \Lambda(N) \rightarrow \mathscr{S}$ is continuous.

Proof. (i) Let $\lambda \in \Lambda(N) \cap \Lambda(\bar{N})$ such that $I(N \times \lambda)=I(\bar{N} \times \lambda)$ and suppose that there exists a sequence $\lambda_{k}$ converging to $\lambda$ with $\lambda_{k} \in \Lambda(N) \cap \Lambda(\bar{N})$ and $I\left(N \times \lambda_{k}\right) \varsubsetneqq I\left(\bar{N} \times \lambda_{k}\right)$. Let $x_{k} \in X$ such that $\left(x_{k}, \lambda_{k}\right) \in I\left(\bar{N} \times \lambda_{k}\right) \backslash I\left(N \times \lambda_{k}\right)$. Let $t_{k} \in \mathbf{R}$ such that $\left(x_{k}, \lambda_{k}\right) \cdot t_{k} \notin N \times \lambda_{k}$. Let $y \in X$ be chosen such that $(y, \lambda)$ is a limit point of $\left(x_{k}, \lambda_{k}\right) \cdot t_{k}$. Then $(y, \lambda) \cdot \mathbf{R} \subset \bar{N} \times \lambda$ and hence $(y, \lambda) \cdot \mathbf{R} \subset N \times \lambda$ since $I(\bar{N} \times \lambda)=I(N \times \lambda)$. But since $\left(x_{k}, \lambda_{k}\right) \cdot t_{k} \notin N \times \lambda_{k}$, we have $y \in N \cap$ $\operatorname{cl}(X \backslash N)$ contradicting $\lambda \in \Lambda(N)$.

(ii) Follows from the definitions.

(iii) If $\bar{N} \subset X$ is compact and $\bar{U} \subset \Lambda(\bar{N})$ open, then

$$
\sigma_{N}^{-1}\left(\sigma_{\bar{N}}(\bar{U})\right)=\bar{U} \cap \Lambda(N, \bar{N})
$$

is open in $\Lambda$. 
The previous lemma shows that the canonical projection map $\pi_{\Lambda}: \mathscr{S} \rightarrow \Lambda$ is a local homeomorphism. If $S \times \lambda \in \mathscr{S}$ and $N \times \lambda$ is an isolating neighborhood for $S \times \lambda$ in $X \times \lambda$, then the map $\sigma_{N}: \Lambda(N) \rightarrow \mathscr{S}$ is an inverse of the restriction of $\pi_{\Lambda}: \mathscr{S} \rightarrow \Lambda$ to the neighborhood $\sigma_{N}(\Lambda(N))$ of $S \times \lambda$ in $\mathscr{S}$.

Remark 6.3. Let $\sigma: \Lambda \rightarrow \mathscr{S}$ be a continuous map with $\pi_{\Lambda}{ }^{\circ} \sigma=1_{\Lambda}$ and let $N \subset X$ be a compact set. Then the set

$$
\begin{aligned}
\Lambda(N, \sigma) & =\sigma^{-1}\left(\sigma_{N}(\Lambda(N))\right)=\{\lambda \in \Lambda(N) \mid \sigma(\lambda)=I(N \times \lambda)\} \\
& =\{\lambda \in \Lambda \mid N \times \lambda \text { is an isolating neighborhood for } \sigma(\lambda) \text { in } X \times \lambda\}
\end{aligned}
$$

is open in $\Lambda$.

Whenever $N \subset X \times \Lambda$ and $K \subset \Lambda$ are compact sets we define

$$
N(K)=N \cap X \times K \text {. }
$$

If $N$ is an isolating neighborhood for the isolated invariant set $S$ in $X \times \Lambda$, then $N(K)$ is an isolating neighborhood for $S(K)$ in $X \times K$.

Lemma 6.4. (i) Let the function $\sigma: \Lambda \rightarrow \mathscr{S}$ satisfy $\pi_{\Lambda} \circ \sigma=1_{\Lambda}$. Then $\sigma$ is continuous if and only if

$$
S=\bigcup_{\lambda \in \Lambda} \sigma(\lambda)
$$

is an isolated invariant set in $X \times \Lambda$.

(ii) Let $\sigma: \Lambda \rightarrow \mathscr{S}$ and $\tau: \Lambda \rightarrow \mathscr{S}$ be continuous functions with $\pi_{\Lambda} \circ \sigma=1_{\Lambda}$ and $\pi_{\Lambda} \circ \tau=1_{\Lambda}$. Then the function $\sigma \cap \tau: \Lambda \rightarrow \mathscr{S}$ which sends $\lambda \in \Lambda$ into $\sigma(\lambda) \cap \tau(\lambda) \in \mathscr{S}$ is continuous.

(iii) Let $\sigma: \Lambda \rightarrow \mathscr{S}$ and $\alpha: \Lambda \rightarrow \mathscr{S}$ be continuous functions such that $\pi_{\Lambda}^{\circ} \sigma=1_{1}$ and $\alpha(\lambda)$ is an attractor in $\sigma(\lambda)$. Furthermore, let $\alpha^{*}(\lambda)$ denote the complementary repeller of $\alpha(\lambda)$ in $\sigma(\lambda)$ and let $S \subset X \times \Lambda$ be defined by (6.1). Then $\alpha^{*}: \Lambda \rightarrow \mathscr{S}$ is continuous and the sets

$$
A=\bigcup_{\lambda \in \Lambda} \alpha(\lambda), \quad A^{*}=\bigcup_{\lambda \in \Lambda} \alpha^{*}(\lambda)
$$

form an attractor-repeller pair in $S$.

Proof. (i) Let us first assume that $S$ is an isolated invariant set, let $N$ be an isolating neighborhood for $S$ in $X \times \Lambda$ and let $N_{0} \times \lambda_{0}$ be an isolating neighborhood for $\sigma\left(\lambda_{0}\right)$ in $X \times \lambda_{0}$ such that $N_{0} \times \lambda_{0} \subset(X \times \Lambda) \backslash \operatorname{cl}((X \times \Lambda) \backslash N)$. Then there exists a compact neighborhood $K_{0}$ of $\lambda_{0}$ in $\Lambda$ such that $N_{0} \times K_{0} \subset N$ and $K_{0} \subset$ $\Lambda\left(N_{0}\right)$. Furthermore we can choose $K_{0}$ small enough such that $S\left(K_{0}\right) \subset N_{0} \times K_{0}$ since otherwise there would exist a sequence $\left(x_{k}, \lambda_{k}\right) \in S$ such that $\lambda_{k}$ converges to $\lambda_{0}$ and $x_{k} \in X \backslash N_{0}$ which would imply that $\left(x_{0}, \lambda_{0}\right) \in \sigma\left(\lambda_{0}\right) \cap \operatorname{cl}\left(X \backslash N_{0} \times \lambda_{0}\right)$ for any limit point $x_{0}$ of $x_{k}$. We conclude that $N_{0} \times \lambda$ is an isolating neighborhood for $\sigma(\lambda)$ in $X \times \lambda$ whenever $\lambda \in K_{0}$ and hence $\sigma(\lambda)=\sigma_{N_{0}}(\lambda)$ for all $\lambda \in K_{0}$. Now the continuity follows from Lemma 6.2(iii).

Conversely suppose that $\sigma: \Lambda \rightarrow \mathscr{S}$ is a continuous function with $\pi_{\Lambda} \circ \sigma=1_{\Lambda}$. Then there exists an isolating neighborhood $N(\lambda) \times \lambda$ for $\sigma(\lambda)$ in $X \times \lambda$ for every $\lambda \in \Lambda$. Furthermore, it follows from Remark 6.3 that the set

$$
\Lambda(N(\lambda), \sigma)=\{\mu \in \Lambda(N(\lambda)) \mid \sigma(\mu)=I(N(\lambda) \times \mu)\}
$$


is open in $\Lambda$ for every $\lambda \in \Lambda$ and hence there exists an open neighborhood $U(\lambda)$ of $\lambda$ in $\Lambda$ such that $N(\lambda) \times \mu$ is an isolating neighborhood for $\sigma(\mu)$ in $X \times \mu$ whenever $\mu \in \operatorname{cl}(U(\lambda))$. Since $\Lambda$ is compact, there exists a finite subcover $U\left(\lambda_{1}\right), \ldots, U\left(\lambda_{n}\right)$ of $\Lambda$. Now define the set

$$
N=\left\{(x, \mu) \in X \times \Lambda \mid \mu \in U\left(\lambda_{j}\right) \Rightarrow x \in N\left(\lambda_{j}\right)\right\} .
$$

Then $N$ is a closed subset of $\bigcup_{j=1}^{n} N\left(\lambda_{j}\right) \times \Lambda$ and therefore $N$ is compact. Moreover, if $(x, \mu) \in S$, then

$$
U=\bigcap_{\mu \notin \operatorname{cl}\left(U\left(\lambda_{j}\right)\right)} \Lambda \backslash U\left(\lambda_{j}\right)
$$

is a neighborhood of $\mu$ in $\Lambda$,

$$
W=\bigcap_{\mu \in \operatorname{cl}\left(U\left(\lambda_{j}\right)\right)} N\left(\lambda_{j}\right)
$$

is a neighborhood of $x$ in $X$ and $W \times U \subset N$. Therefore $N$ is a neighborhood of $S$ in $X \times \Lambda$. Finally, $S=I(N)$ since $(x, \mu) \cdot \mathbf{R} \subset N$ and $\mu \in U\left(\lambda_{j}\right)$ imply that $(x, \mu) \cdot \mathbf{R}$ $\subset N\left(\lambda_{j}\right) \times \mu$ and thus $(x, \mu) \in \sigma(\mu)$.

(ii) Choose compact sets $N_{1} \subset X$ and $N_{2} \subset X$ such that $N_{1} \times \lambda_{0}$ and $N_{2} \times \lambda_{0}$ are isolating neighborhoods for $\sigma\left(\lambda_{0}\right)$ and $\tau\left(\lambda_{0}\right)$, respectively, in $X \times \lambda_{0}$. By Remark 6.3, there exists a compact neighborhood $K_{0}$ of $\lambda_{0}$ in $\Lambda$ such that $K_{0} \subset \Lambda\left(N_{1}, \sigma\right) \cap$ $\Lambda\left(N_{2}, \tau\right)$. Hence $N_{1} \cap N_{2} \times \lambda$ is an isolating neighborhood for $\sigma(\lambda) \cap \tau(\lambda)$ in $X \times \lambda$ whenever $\lambda \in K_{0}$. This implies that $\sigma \cap \tau(\lambda)=\sigma_{N_{1} \cap N_{2}}(\lambda)$ for every $\lambda \in K_{0}$ and thus the continuity follows from Lemma 6.2.

(iii) It follows from (i) that $A$ is a compact subset of $S$. We show that $\operatorname{cl}\left(A^{*}\right) \cap A$ $=\varnothing$. For this purpose let $N \times \lambda$ be an isolating neighborhood of $\alpha(\lambda)$ in $X \times \lambda$ with $N \times \lambda \cap \alpha^{*}(\lambda)=\varnothing$. Suppose that there exists a sequence $\left(x_{k}, \lambda_{k}\right) \in \alpha^{*}\left(\lambda_{k}\right)$ converging to $(x, \lambda) \in \alpha(\lambda)$ with $x_{k} \in N, \lambda_{k} \in \Lambda(N, \alpha)$. Then $\omega\left(x_{k}, \lambda_{k}\right) \cap \alpha\left(\lambda_{k}\right)$ $=\varnothing$ and hence $\left(x_{k}, \lambda_{k}\right) \cdot[0, \infty) \not \subset N \times \lambda_{k}$. Let $t_{k}>0$ be the maximal time with $\left(x_{k}, \lambda_{k}\right) \cdot\left[0, t_{k}\right] \subset N \times \lambda_{k}$ and let $(y, \lambda) \in \sigma(\lambda)$ be any limit point of $\left(x_{k}, \lambda_{k}\right) \cdot t_{k}$. Then $y \notin$ int $N$ and hence $(y, \lambda) \notin \alpha(\lambda)$. But if the sequence $t_{k}$ is bounded then $(y, \lambda) \in(x, \lambda) \cdot \mathbf{R} \subset \alpha(\lambda)$ and if the sequence is unbounded then $(y, \lambda) \cdot(-\infty, 0]$ $\subset N \times \lambda$ and therefore $(y, \lambda) \in \omega(N \times \lambda \cap \sigma(\lambda))=\alpha(\lambda)$, a contradiction. We conclude that $\operatorname{cl}\left(A^{*}\right) \cap A=\varnothing$. Hence there exists a neighborhood $U$ of $A$ in $S$ such that $\operatorname{cl}(U) \cap A^{*}=\varnothing$. If $(x, \lambda) \cdot(-\infty, 0] \subset U$, then $(x, \lambda) \in \sigma(\lambda)$ and $\omega^{*}(x, \lambda) \cap$ $\alpha^{*}(\lambda)=\varnothing$ and therefore $(x, \lambda) \in \alpha(\lambda) \subset A$ (Lemma 3.2). By Lemma 3.1, this implies that $A$ is an attractor in $S$. It follows again from Lemma 3.2 that its complementary repeller is given by

$$
A^{*}=\bigcup_{\lambda \in \Lambda} \alpha^{*}(\lambda)=\{(x, \lambda) \in S \mid \omega(x, \lambda) \cap A=\varnothing\} .
$$

In some situations it might be useful to consider general maps $\tau: \Delta \rightarrow \mathscr{S}$ such that $\pi_{\Lambda} \circ \tau: \Delta \rightarrow \Lambda$ is not necessarily injective. This can be reformulated within the framework of this section by considering $X \times \Delta$ as a local flow in $\Gamma \times \Delta$, where $(x, \xi) \in X \times \Delta$ is identified with the triple $\left(x, \pi_{\Lambda} \circ \tau(\xi), \xi\right) \in X \times \Lambda \times \Delta \subset \Gamma \times \Delta$. 


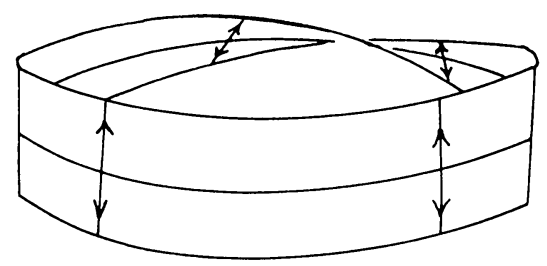

Figure 5

Introducing the space

$$
\mathscr{S}_{\Delta}=\left\{S \times \lambda \times \xi \mid \xi \in \Delta, S \times \lambda \in \mathscr{S}, \lambda=\pi_{\Delta} \circ \tau(\xi)\right\}
$$

endowed with an analogous topology as $\mathscr{S}$, it is then easy to see that the map $\tau_{\Delta}: \Delta \rightarrow \mathscr{S}_{\Delta}$ defined by $\tau_{\Delta}(\xi)=\tau(\xi) \times \xi$ for $\xi \in \Delta$ is continuous.

At the end of this section we point out that some (global) phenomena cannot be described within the framework of product flows $X \times \Lambda$. An example for such a situation is the flow on a Moebius strip which is illustrated in Figure 5 and involves a change of orientation in the space $X$. In such cases it might be useful to consider a local flow on a (locally trivial) fibration instead of the product space $X \times \Lambda$. This could be a problem for future investigations.

6.2. Local continuation. In this section we are going to prove a local continuation theorem for the Conley index of isolated invariant sets. The result has been formulated in Conley [3] but the proof is only roughly sketched. A complete but rather complicated proof can be found in Kurland [8]. We present a simplified proof which is based on the results in \$4.2.

Throughout this section we will adopt the notation of $\S 6.1$ and assume in addition that $\sigma: \Lambda \rightarrow \mathscr{S}$ is a continuous map with $\pi_{\Lambda} \circ \sigma=1_{\Lambda}$ and that the isolated invariant set $S$ in $X \times \Lambda$ is defined by (6.1). Note that for any index pair $\left(N_{1}, N_{0}\right)$ for $S$ in $X \times \Lambda$ and for any compact set $K \subset \Lambda$ the sets $\left(N_{1}(K), N_{0}(K)\right)$ form an index pair for the isolated invariant set $S(K)$ in $X \times K$.

The local continuation theorem now consists of two parts. The first and easy part is to show that for any index pair $\left(N_{1}, N_{0}\right)$ for $S$ in $X \times \Lambda$ the canonical injection map $j(\lambda): N_{1}(\lambda) / N_{0}(\lambda) \rightarrow N_{1} / N_{0}$ induces a morphism between the corresponding connected simple systems which is independent of the choice of the index pair (Proposition 6.5). The main part is then to show that this morphism is in fact a local isomorphism (Theorem 6.7). These facts will then be used to obtain a continuation result for the coexact sequence of $\$ 5$ which is associated with an attractor-repeller pair in $S(\$ 6.4)$.

Proposition 6.5. Let $\left(N_{1}, N_{0}\right)$ be an index pair for $S$ in $X \times \Lambda$. Then the canonical injection map $j(\lambda): N_{1}(\lambda) / N_{0}(\lambda) \rightarrow N_{1} / N_{0}$ induces a morphism between the connected simple systems $I(\sigma(\lambda), X \times \lambda)$ and $I(S, X \times \Lambda)$ which is independent of the choice of the index pair. 
Proof. Let $\left(\bar{N}_{1}, \bar{N}_{0}\right)$ be another index pair for $S$ in $X \times \Lambda$, choose $T>0$ such that

$$
\begin{gathered}
(x, \mu) \cdot[-T, T] \subset N_{1} \backslash N_{0} \Rightarrow(x, \mu) \in \bar{N}_{1} \backslash \bar{N}_{0}, \\
(x, \mu) \cdot[-T, T] \subset \bar{N}_{1} \backslash \bar{N}_{0} \Rightarrow(x, \mu) \in N_{1} \backslash N_{0}
\end{gathered}
$$

and define the maps $f(\lambda)^{t}: N_{1}(\lambda) / N_{0}(\lambda) \rightarrow \bar{N}_{1}(\lambda) / \bar{N}_{0}(\lambda)$ and $g^{t}: \bar{N}_{1} / \bar{N}_{0} \rightarrow N_{1} / N_{0}$ by

$$
\begin{aligned}
& f(\lambda)^{t}[x, \lambda] \\
&= \begin{cases}{[(x, \lambda) \cdot 3 t],} & (x, \lambda) \cdot[0,2 t] \subset N_{1} \backslash N_{0},(x, \lambda) \cdot[t, 3 t] \subset \bar{N}_{1} \backslash \bar{N}_{0}, \\
{\left[\bar{N}_{0}(\lambda)\right],} & \text { otherwise },(x, \lambda) \in N_{1}(\lambda),\end{cases} \\
& g^{t}[x, \mu]= \begin{cases}{[(x, \mu) \cdot 3 t],} & (x, \mu) \cdot[0,2 t] \subset \bar{N}_{1} \backslash \bar{N}_{0},(x, \mu) \cdot[t, 3 t] \subset N_{1} \backslash N_{0}, \\
{\left[N_{0}\right],} & \text { otherwise, }(x, \mu) \in \bar{N}_{1},\end{cases}
\end{aligned}
$$

for $t \geqslant T$. Then the composed map $g^{t} \circ \bar{j}(\lambda) \circ f(\lambda)^{t}: N_{1}(\lambda) / N_{0}(\lambda) \rightarrow N_{1} / N_{0}$ is given by

$$
g^{t} \circ \bar{j}(\lambda) \circ f(\lambda)^{t}[x, \lambda]= \begin{cases}{[(x, \lambda) \cdot 6 t],} & (x, \lambda) \cdot[0,6 t] \subset N_{1} \backslash N_{0}, \\ {\left[N_{0}\right],} & \text { otherwise, }\end{cases}
$$

for $(x, \lambda) \in N_{1}(\lambda)$ and hence is homotopic to the injection $j(\lambda): N_{1}(\lambda) / N_{0}(\lambda) \rightarrow$ $N_{1} / N_{0}$.

The next lemma provides the crucial step in proving that the injection map $j(\lambda): N_{1}(\lambda) / N_{0}(\lambda) \rightarrow N_{1} / N_{0}$ is (locally) a homotopy equivalence.

Lemma 6.6. Let $\left(N_{1}, N_{0}\right)$ be an index pair for $S$ in $X \times \Lambda$ and let $\lambda_{0} \in \Lambda$ be given. Then there exists a compact neighborhood $K$ of $\lambda_{0}$ in $\Lambda$ and times $T_{1}>2 T_{0}>0$ such that the following statements hold.

(i) $N \times K$ is an isolating neighborhood of $S(K)$ and $U \times K$ is a neighborhood of $S(K)$ in $X \times K$, where the sets $N \subset X$ and $U \subset X$ are defined by

$$
\begin{gathered}
N=\operatorname{cl}\left(\pi_{X}\left(N_{1}(K) \backslash N_{0}(K)\right)\right)=\pi_{X}\left(\operatorname{cl}\left(N_{1}(K) \backslash N_{0}(K)\right)\right), \\
U=\operatorname{int} \bigcap_{\lambda \in K} \pi_{X}\left(N_{1}(\lambda) \backslash N_{0}(\lambda)\right) \text { rel } X .
\end{gathered}
$$

(ii) For all $x \in X$ and $\mu, \lambda \in K$ and $T \geqslant T_{1}$ the following implications hold.

$$
\begin{gathered}
(x, \mu) \cdot\left[-T_{0}, T_{0}\right] \subset N \times K \Rightarrow x \in U, \\
\left(\pi_{X}((x, \mu) \cdot[0, T]), \lambda\right) \subset \operatorname{cl}\left(N_{1}(K) \backslash N_{0}(K)\right), \\
(x, \mu) \cdot\left[T_{0}, T\right] \not \subset U \times K \Rightarrow\left(\pi_{X}((x, \mu) \cdot T), \lambda\right) \cdot[0, T] \cap N_{0} \neq \varnothing .
\end{gathered}
$$

Proof. In order to prove statement (i) let us choose isolating neighborhoods $\underline{N}$ and $\bar{N}$ for $S$ in $X \times \Lambda$ such that $N_{1} \backslash N_{0}$ is a neighborhood of $\underline{N}$ in $X \times \Lambda$ and $\bar{N}$ is a neighborhood of $\mathrm{cl}\left(N_{1} \backslash N_{0}\right)$ in $X \times \Lambda$ (Lemma 3.7). By Remark 6.3, there exists a compact neighborhood $K$ of $\lambda_{0}$ in $\Lambda$ such that

$$
K \subset \Lambda\left(\pi_{X}\left(\underline{N}\left(\lambda_{0}\right)\right), \sigma\right) \cap \Lambda\left(\pi_{X}\left(\bar{N}\left(\lambda_{0}\right)\right), \sigma\right) .
$$


This implies that

$$
\pi_{X}\left(\underline{N}\left(\lambda_{0}\right)\right) \times K, \quad \pi_{X}\left(\bar{N}\left(\lambda_{0}\right)\right) \times K
$$

are isolating neighborhoods for $S(K)$ in $X \times K$. Furthermore we can choose $K$ small enough such that

$$
\begin{gathered}
\pi_{X}\left(\underline{N}\left(\lambda_{0}\right)\right) \times K \subset N_{1} \backslash N_{0}, \\
\pi_{X}\left(N_{1}(\lambda) \backslash N_{0}(\lambda)\right) \subset \pi_{X}\left(\bar{N}\left(\lambda_{0}\right)\right) \quad \forall \lambda \in K .
\end{gathered}
$$

The first inclusion is obvious since $\underline{N}\left(\lambda_{0}\right)$ is contained in the interior of $N_{1} \backslash N_{0}$ relative $X \times \Lambda$. If (6.8) would not hold for any neighborhood $K$ of $\lambda_{0}$, then there would exist a sequence $\left(x_{k}, \lambda_{k}\right) \in N_{1} \backslash N_{0}$ such that $\lambda_{k}$ tends to $\lambda_{0}$ and $\left(x_{k}, \lambda_{0}\right) \notin \bar{N}$. But then any limit point $x_{0}$ of $x_{k}$ would satisfy $\left(x_{0}, \lambda_{0}\right) \in \operatorname{cl}\left(N_{1} \backslash N_{0}\right)$, and $\left(x_{0}, \lambda_{0}\right)$ $\in \operatorname{cl}((X \times \Lambda) \backslash \bar{N})$, contradicting the fact that $\bar{N}$ is a neighborhood of $\operatorname{cl}\left(N_{1} \backslash N_{0}\right)$ in $X \times \Lambda$. From (6.7) and (6.8) we conclude that

$$
\pi_{X}\left(\underline{N}\left(\lambda_{0}\right)\right) \subset \pi_{X}\left(N_{1}(\lambda) \backslash N_{0}(\lambda)\right) \subset \pi_{X}\left(\bar{N}\left(\lambda_{0}\right)\right)
$$

for all $\lambda \in K$. This proves statement (i).

In order to prove statement (ii), let us choose the compact neighborhood $K$ of $\lambda_{0}$ in $\Lambda$ as in (i). Then it follows from Lemma 4.6 that (6.5) holds for some $T_{0}>0$. Furthermore note that once (6.6) is satisfied for some $T=T_{1}>2 T_{0}$, then it holds for all $T \geqslant T_{1}$ since the first condition in (6.6) together with (6.5) guarantees that $(x, \mu) \cdot\left[T_{0}, T-T_{0}\right] \subset U \times K$. Now suppose that (6.6) does not hold for any $T=T_{1}$ $>0$ and any neighborhood of $\lambda_{0}$ in $\Lambda$. Then there would exist sequences $x_{k} \in X$, $\lambda_{k} \in K, \mu_{k} \in K, T_{k}>2 T_{0}, t_{k} \in\left[T_{0}, T_{k}\right]$ such that $T_{k}$ tends to infinity, $\lambda_{k}$ and $\mu_{k}$ tend to $\lambda_{0}$ and

$$
\begin{gathered}
\left(\pi_{X}\left(\left(x_{k}, \mu_{k}\right) \cdot\left[0, T_{k}\right]\right), \lambda_{k}\right) \subset \operatorname{cl}\left(N_{1}(K) \backslash N_{0}(K)\right), \\
\left(x_{k}, \mu_{k}\right) \cdot t_{k} \notin U \times K, \\
\left(\pi_{X}\left(\left(x_{k}, \mu_{k}\right) \cdot T_{k}\right), \lambda_{k}\right) \cdot\left[0, T_{k}\right] \subset N_{1} \backslash N_{0} .
\end{gathered}
$$

It follows from (6.9) and (6.5) that $\left(x_{k}, \mu_{k}\right) \cdot\left[T_{0}, T_{k}-T_{0}\right] \subset U \times K$ and therefore $t_{k} \geqslant T_{k}-T_{0}$. Now let $x_{0} \in N$ be a limit point of $\pi_{X}\left(\left(x_{k}, \mu_{k}\right) \cdot T_{k}\right)$ and let $\tau \geqslant 0$ be a limit point of $T_{k}-t_{k}$. Then it follows from (6.9) and (6.11) that $\left(x_{0}, \lambda_{0}\right) \cdot \mathbf{R} \subset$ $\operatorname{cl}\left(N_{1} \backslash N_{0}\right)$ and hence $\left(x_{0}, \lambda_{0}\right) \in S$. But it follows from (6.10) that $\left(x_{0}, \lambda_{0}\right) \cdot(-\tau) \notin$ $U \times K$, contradicting the fact that $S(K) \subset U \times K$.

THEOREM 6.7. Let $\left(N_{1}, N_{0}\right)$ be an index pair for $S$ in $X \times \Lambda$, let $\lambda_{0} \in \Lambda$ be given and let $K$ be a compact, contractible neighborhood of $\lambda_{0}$ in $\Lambda$ which satisfies the conditions of Lemma 6.6 for $T_{1}>2 T_{0}>0$. Then the injection map

$$
j(\lambda)=j_{K}(\lambda): N_{1}(\lambda) / N_{0}(\lambda) \rightarrow N_{1}(K) / N_{0}(K)
$$


is a homotopy equivalence for every $\lambda \in K$ and the map $f(\lambda)=f_{K}(\lambda): N_{1}(K) / N_{0}(K)$ $\rightarrow N_{1}(\lambda) / N_{0}(\lambda)$, defined by

(6.12) $f_{K}(\lambda)[x, \mu]$

$$
=\left\{\begin{array}{l}
{\left[\left(\pi_{X}((x, \mu) \cdot 3 T), \lambda\right) \cdot T\right], \quad \text { if }(x, \mu) \cdot[0,2 T] \subset N_{1} \backslash N_{0},} \\
\quad\left(\pi_{X}((x, \mu) \cdot[T, 3 T]), \lambda\right) \subset N_{1} \backslash N_{0}, \\
\quad\left(\pi_{X}((x, \mu) \cdot 3 T), \lambda\right) \cdot[0, T] \subset N_{1} \backslash N_{0}, \\
{\left[N_{0}(\lambda)\right], \text { otherwise }}
\end{array}\right.
$$

for $(x, \mu) \in N_{1}(K)$, is a homotopy inverse of $j(\lambda)$.

Proof. First note that the composed map $f(\lambda) \circ j(\lambda): N_{1}(\lambda) / N_{0}(\lambda) \rightarrow$ $N_{1}(\lambda) / N_{0}(\lambda)$ is given by

$$
f(\lambda) \circ j(\lambda)[x, \lambda]= \begin{cases}{[(x, \lambda) \cdot 4 T],} & \text { if }(x, \lambda) \cdot[0,4 T] \subset N_{1} \backslash N_{0}, \\ {\left[N_{0}(\lambda)\right],} & \text { otherwise, }\end{cases}
$$

for $(x, \lambda) \in N_{1}(\lambda)$ and it follows from Lemma 4.7 that this map is homotopic to the identity. In order to show that $j(\lambda) \circ f(\lambda)$ is also homotopic to the identity we make use of the fact that $K$ is contractible which means that there exists a function $r: K \times[0,1] \rightarrow K$ such that

$$
r(\mu, 0)=\mu, \quad r(\mu, 1)=\lambda \quad \forall \mu \in K .
$$

Now we define the map $F: N_{1}(K) / N_{0}(K) \times[0,1] \rightarrow N_{1}(K) / N_{0}(K)$ by

$$
F([x, \mu], \xi)=\left\{\begin{aligned}
& {[}\left.\left(\pi_{X}((x, \mu) \cdot 3 T), r(\mu, \xi)\right) \cdot T\right], \quad \text { if }(x, \mu) \cdot[0,2 T] \subset N_{1} \backslash N_{0}, \\
&\left(\pi_{X}((x, \mu) \cdot[T, 3 T]), r(\mu, \xi)\right) \subset N_{1} \backslash N_{0}, \\
&\left(\pi_{x}((x, \mu) \cdot 3 T), r(\mu, \xi)\right) \cdot[0, T] \subset N_{1} \backslash N_{0}, \\
& {\left[N_{0}(K)\right], \text { otherwise, } }
\end{aligned}\right.
$$

for $(x, \mu) \in N_{1}(K)$ and $\xi \in[0,1]$. Then it follows from (6.13) and (6.12) that $F(\cdot, 1)=j(\lambda) \circ f(\lambda)$ and that $F(\cdot, 0): N_{1}(K) / N_{0}(K) \rightarrow N_{1}(K) / N_{0}(K)$ is given by

$$
F([x, \mu], 0)= \begin{cases}{[(x, \mu) \cdot 4 T],} & \text { if }(x, \mu) \cdot[0,4 T] \subset N_{1} \backslash N_{0}, \\ {\left[N_{0}(K)\right],} & \text { otherwise, }\end{cases}
$$

for $(x, \mu) \in N_{1}(K)$ and therefore is homotopic to the identity (Lemma 4.7). It remains to show that $F$ is continuous.

In the following cases the continuity can be obtained by standard arguments as in the proof of Lemma 4.7 or Theorem 5.7(iii).

1. $(x, \mu) \cdot[0,2 T] \not \subset \mathrm{cl}\left(N_{1}(K) \backslash N_{0}(K)\right)$,

2. $\left(\pi_{X}((x, \mu) \cdot[T, 3 T]), r(\mu, \xi)\right) \not \subset \operatorname{cl}\left(N_{1}(K) \backslash N_{0}(K)\right)$,

3. $\left(\pi_{X}((x, \mu) \cdot 3 T), r(\mu, \xi)\right) \cdot[0, T] \not \subset \mathrm{cl}\left(N_{1}(K) \backslash N_{0}(K)\right)$,

4. $\left(\pi_{X}((x, \mu) \cdot 3 T), r(\mu, \xi)\right) \cdot T \in N_{0}$.

Therefore we can assume from now on that

$$
\begin{gathered}
(x, \mu) \cdot[0,2 T] \subset \operatorname{cl}\left(N_{1}(K) \backslash N_{0}(K)\right), \\
\left(\pi_{X}((x, \mu) \cdot[T, 3 T]), r(\mu, \xi)\right) \subset \operatorname{cl}\left(N_{1}(K) \backslash N_{0}(K)\right), \\
\left(\pi_{X}((x, \mu) \cdot 3 T), r(\mu, \xi)\right) \cdot[0, T] \subset N_{1} \backslash N_{0} .
\end{gathered}
$$




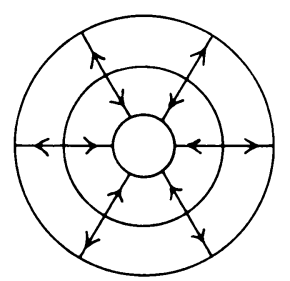

Figure 6

Now Lemma 6.7 comes in. First of all, since $T \geqslant 2 T_{0}$ it follows from (6.14) and (6.5) that $(x, \mu) \cdot\left[T, T+T_{0}\right] \subset U \times K$, where $U \subset X$ is defined by (6.4). Secondly, it follows from (6.15), (6.16) and (6.6) that $(x, \mu) \cdot\left[T+T_{0}, 3 T\right] \subset U \times K$. In particu$\operatorname{lar}(x, \mu) \cdot 2 T \notin N_{0}$. Therefore the conditions (6.14) and (6.15) can be replaced by

$$
\begin{aligned}
& (x, \mu) \cdot[0,2 T] \subset N_{1} \backslash N_{0}, \\
& (x, \mu)^{\prime} \cdot[T, 3 T] \subset U \times K .
\end{aligned}
$$

But the conditions (6.16), (6.17) and (6.18) together are stable with respect to small variations in $(x, \mu) \in N_{1}(K)$ and $\xi \in[0,1]$. This proves the continuity of $F$.

Since $\Lambda$ is a connected space, we obtain as an immediate consequence of Theorem 6.7 that the homotopy index $h(\sigma(\lambda))$ of the isolated invariant set $\sigma(\lambda)$ in $X \times \lambda$ is independent of $\lambda$. In fact, it depends only on the path-component of $\sigma(\lambda)$ in $\mathscr{S}$ (Lemma 6.4 together with Theorem 6.7). Furthermore, combining Theorem 6.7 with Proposition 6.5 , we obtain that there is locally a unique isomorphism between the connected simple systems $I(\sigma(\lambda), X \times \lambda)$ and $I(\sigma(\mu), X \times \mu)$. If $\Lambda$ is connected, then such an isomorphism exists for any two $\lambda, \mu \in \Lambda$. However, we will see in the next section that globally this isomorphism need no longer be unique.

6.3. Global continuation. We first point out that the global isolated invariant set $S$ in $X \times \Lambda$ may have a much richer structure than the isolated invariant set $\sigma(\lambda)$ in a single fiber $X \times \lambda$. This is illustrated by Figure 6 in which the flow on $\Gamma=X \times \Lambda$ $=\mathbf{R} \times S^{1}$ is given by $(x, \lambda) \cdot t=\left(x e^{t}, \lambda\right)$ for $x \in \mathbf{R}$ and $\lambda \in S^{1}$. Then the homotopy index of $\sigma(\lambda)=(0, \lambda)$ is $h(\sigma(\lambda))=\Sigma^{1}$ but $h(S)=\Sigma^{2} \vee \Sigma^{1}$. Hence the global injection $j(\lambda): N_{1}(\lambda) / N_{0}(\lambda) \rightarrow N_{1} / N_{0}$ cannot be a homotopy equivalence in this case. However, if $\Lambda$ is a contractible space, one might expect that this global injection is indeed a homotopy equivalence. We leave this as an open problem.

In order to obtain an isomorphism between any two connected simple systems $I(\sigma(\lambda), X \times \lambda)$ and $I(\sigma(\mu), X \times \mu)$ it is useful to rephrase the statement of Theorem 6.7 as follows.

Corollary 6.8. Let $\left(N_{1}, N_{0}\right)$ be an index pair for $S$ in $X \times \Lambda$ and let $K \subset \Lambda$ be a compact, contractible set which satisfies the conditions of Lemma 6.6 for $T_{1}>2 T_{0}>0$. For $\lambda, \mu \in K$ and $T \geqslant T_{1}$ let the map $F(\lambda, \mu)=F_{K}(\lambda, \mu): N_{1}(\mu) / N_{0}(\mu) \rightarrow$ $N_{1}(\lambda) / N_{0}(\lambda)$ be defined by

$$
F_{K}(\lambda, \mu)[x, \mu]=\left\{\begin{array}{c}
{\left[\left(\pi_{X}((x, \mu) \cdot 3 T), \lambda\right) \cdot T\right], \quad \text { if }(x, \mu) \cdot[0,2 T] \subset N_{1} \backslash N_{0},} \\
\quad\left(\pi_{X}((x, \mu) \cdot[T, 3 T]), \lambda\right) \subset N_{1} \backslash N_{0}, \\
\left(\pi_{X}((x, \mu) \cdot 3 T), \lambda\right) \cdot[0, T] \subset N_{1} \backslash N_{0}, \\
{\left[N_{0}(\lambda)\right], \text { otherwise, }}
\end{array}\right.
$$


for $(x, \mu) \in N_{1}(\mu)$. Then for all $\lambda, \mu, \nu \in K$

$$
\begin{gathered}
F_{K}(\lambda, \mu) \circ F_{K}(\mu, \nu) \sim F_{K}(\lambda, \nu), \\
F_{K}(\lambda, \lambda) \sim 1 .
\end{gathered}
$$

In particular, $F_{K}(\lambda, \mu)$ is a homotopy equivalence with homotopy inverse $F_{K}(\mu, \lambda)$.

Furthermore, if $\left(\bar{N}_{1}, \bar{N}_{0}\right)$ is another index pair for $S$ in $X \times \Lambda$ with respect to which $K$ satisfies the conditions of Lemma 6.6 and if $\bar{F}_{K}(\lambda, \mu): \bar{N}_{1}(\mu) / \bar{N}_{0}(\mu) \rightarrow \bar{N}_{1}(\lambda) / \bar{N}_{0}(\lambda)$ is defined analogously to (6.19), then the maps $\bar{F}_{K}(\lambda, \mu)$ and $F_{K}(\lambda, \mu)$ induce the same isomorphism between the connected simple systems $I(\sigma(\mu), X \times \mu)$ and $I(\sigma(\lambda), X \times \lambda)$.

Proof. With the notation of Theorem 6.7 we have $F_{K}(\lambda, \mu)=f_{K}(\lambda) \circ j_{K}(\mu)$. Therefore (6.20) and (6.21) follow from the fact that $f_{K}(\lambda) \circ j_{K}(\lambda)$ and $j_{K}(\lambda) \circ f_{K}(\lambda)$ are homotopic to the respective identity maps for every $\lambda \in K$. The remainder of the corollary is a consequence of Proposition 6.5.

If $\Lambda$ is a compact, connected space we can connect any two points $\mu \in \Lambda$ and $\lambda \in \Lambda$ by a finite sequence of compact sets $K$ each of which satisfies the conditions of Lemma 6.6. Any such sequence induces an isomorphism between the connected simple systems $I(\sigma(\mu), X \times \mu)$ and $I(\sigma(\lambda), X \times \lambda)$. This motivates the introduction of the following subcategory of pointed spaces and homotopy classes of maps associated with a continuous function $\sigma: \Lambda \rightarrow \mathscr{S}_{\text {satisfying }} \pi_{\Lambda}{ }^{\circ} \sigma=1_{\Lambda}$. This category may be considered as the global Conley index of $\sigma$ in $X \times \Lambda$ and is defined by

$$
I(\sigma, X, \Lambda)=\left(I_{0}, I_{m}\right),
$$

where

$(6.22 ; 2) \quad I_{0}=\left\{N_{1}(\lambda) / N_{0}(\lambda) \mid \lambda \in \Lambda\right.$ and $N_{1}(\lambda), N_{0}(\lambda)$ is an index

$$
\begin{gathered}
\text { pair for } \sigma(\lambda) \text { in } X \times \lambda\} \\
I_{m}=\left\{[f] \mid N_{1}(\lambda) / N_{0}(\lambda) \in I_{0}, N_{1}(\mu) / N_{0}(\mu) \in I_{0}\right. \text { and } \\
f: N_{1}(\mu) / N_{0}(\mu) \rightarrow N_{1}(\lambda) / N_{0}(\lambda) \text { is a finite composition } \\
\text { of maps defined in Lemma } 4.7 \text { and Corollary 6.8 }\} .
\end{gathered}
$$

The global Conley index $I(\sigma, X, \Lambda)$ of $\sigma$ in $X \times \Lambda$ has to be well distinguished from the Conley index $I(S, X \times \Lambda)$ of the global isolated invariant set $S$ in $X \times \Lambda$. If $\Lambda$ is connected, then the category $I(\sigma, X, \Lambda)$ has the property that there is at least one morphism between any two objects and that every morphism is an equivalence. However, $I(\sigma, X, \Lambda)$ is in general not a connected simple system since there may be different isomorphisms between the same objects. This is the case in the parametrized flow illustrated in Figure 7. However, if $\Lambda$ is simply connected (every closed arc is homotopic to a constant where the end points are fixed), then we will show that $I(\sigma, X, \Lambda)$ is a connected simple system. This result has first been stated in terms of the cohomology of the isolated invariant sets and is due to Montgomery [9]. The corresponding theorem in Conley [3] has been phrased in terms of continuation along arcs. 


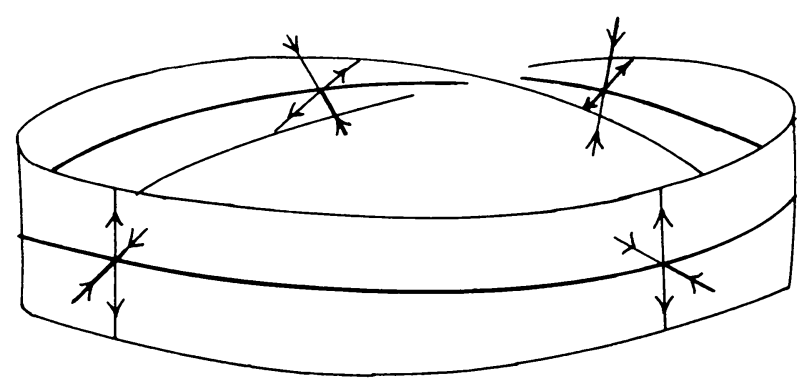

FIGURE 7

THEOREM 6.9. Suppose that $\Lambda$ is simply connected and let $\sigma: \Lambda \rightarrow \mathscr{S}$ be a continuous map with $\pi_{\Lambda} \circ \sigma=1_{\Lambda}$. Then $I(\sigma, X, \Lambda)$ is a connected simple system.

Proof. We have to prove that every morphism in $I_{m}$ with the same domain and range is in the class of the identity map. Using the second part of Corollary 6.8 one can first show that every map $f: N_{1}(\lambda) / N_{0}(\lambda) \rightarrow N_{1}(\lambda) / N_{0}(\lambda)$ with $[f] \in I_{m}$ is homotopic to a finite composition of maps defined by (6.19) and associated with a single index pair $\left(N_{1}, N_{0}\right)$ for $S$ in $X \times \Lambda$. Hence we can assume that there are finitely many compact contractible sets $K_{0}, \ldots, K_{k}$ in $\Lambda$ satisfying the conditions of Lemma 6.6 and points $\lambda_{j} \in K_{j-1} \cap K_{j}, j=1, \ldots, k, \lambda_{0}=\lambda_{k+1}=\lambda \in K_{0} \cap K_{k}$ such that

$$
f=F_{k}\left(\lambda_{k+1}, \lambda_{k}\right) \circ F_{k-1}\left(\lambda_{k}, \lambda_{k-1}\right) \circ \cdots \circ F_{0}\left(\lambda_{1}, \lambda_{0}\right),
$$

where the maps $F_{j}=F_{K_{j}}$ are defined by (6.19). Let us extend $\left\{K_{0}, \ldots, K_{k}\right\}$ to a collection of compact, contractible sets $K_{0}, \ldots, K_{n}$ which satisfy the conditions of Lemma 6.6 for $T \geqslant T_{1}>2 T_{0}>0$ and are chosen such that the sets int $K_{j}=$ $\Lambda \backslash \operatorname{cl}\left(\Lambda \backslash K_{j}\right)$ cover $\Lambda$. Using (6.20) we can assume without loss of generality that $\lambda_{j} \in$ int $K_{j-1} \cap$ int $K_{j}$ for $j=1, \ldots, k$ and $\lambda_{0} \in$ int $K_{0} \cap$ int $K_{k}$. Since the sets $K_{j}$ are contractible, there exists an arc $\alpha_{1}:[0,1] \rightarrow \bigcup_{j=0}^{k}$ int $K_{j}$ such that $\alpha_{1}(0)=\alpha_{1}(1)=$ $\lambda_{0}$ and $\lambda_{j}=\alpha_{1}\left(\xi_{j}\right), \alpha_{1}\left(\left[\xi_{j}, \xi_{j+1}\right]\right) \subset$ int $K_{j}$ for $j=0, \ldots, k$. Now we make use of the fact that $\Lambda$ is simply connected and conclude that there exists a continuous family of arcs $\alpha_{t}:[0,1] \rightarrow \Lambda, 0 \leqslant t \leqslant 1$, such that $\alpha_{t}(0)=\alpha_{t}(1)=\lambda_{0}$ for all $t \in[0,1]$ and $\alpha_{0}(\xi) \equiv \lambda_{0}$. With every arc $\alpha_{t}$ we can associate a sequence of maps $F_{\nu(j)}\left(\mu_{j+1}, \mu_{j}\right)$, $j=0, \ldots, l$, defined by (6.19) and such that $\mu_{j}=\alpha_{t}\left(\zeta_{j}\right), 0=\zeta_{0}<\zeta_{1}<\cdots<\zeta_{l+1}$ $=1$, and $\alpha_{t}\left(\left[\zeta_{j}, \zeta_{j+1}\right]\right) \subset$ int $K_{\nu(j)}, j=0, \ldots, l$. Let $f_{t}: N_{1}(\lambda) / N_{0}(\lambda) \rightarrow N_{1}(\lambda) / N_{0}(\lambda)$ denote the composition of these maps. Then it follows from (6.20) that the homotopy class of $f_{t}$ is independent of the choice of the points $\zeta_{j}$ and the indices $\nu(j)$. This fact together with the continuous dependence of the condition $\alpha_{t}\left(\left[\zeta_{j}, \zeta_{j+1}\right]\right) \subset$ int $K_{\nu(j)}$ on $t$ shows that $\left[f_{t}\right]$ is independent of $t \in[0,1]$. Finally, it follows from (6.20) and (6.21) that $f_{0}$ is homotopic to the identity on $N_{1}(\lambda) / N_{0}(\lambda)$.

6.4. Continuation of attractor-repeller pairs. It is the purpose of this section to combine the continuation results of $\S \S 6.1-6.3$ with the coexact sequence of $\S 5$ associated with an attractor-repeller pair. 
In addition to the notation and assumptions of $\S 6.1$ we will assume throughout this section that $\sigma: \Lambda \rightarrow \mathscr{S}$ and $\alpha: \Lambda \rightarrow \mathscr{S}$ are continuous maps such that $\pi_{\Lambda}{ }^{\circ} \sigma=1_{\Lambda}$ and $\alpha(\lambda)$ is an attractor in $\sigma(\lambda)$. Then the complementary repeller $\alpha^{*}(\lambda)$ of $\alpha(\lambda)$ in $\sigma(\lambda)$ also defines a continuous function from $\Lambda$ into $\mathscr{S}$ and the sets $A, A^{*}$ defined by (6.2) form an attractor-repeller pair for the isolated invariant set $S$ defined by (6.1) in $X \times \Lambda$ (Lemma 6.4).

Now let $N_{0} \subset N_{1} \subset N_{2}$ be a filtration of compact sets in $X \times \Lambda$ such that $\left(N_{2}, N_{0}\right)$ and $\left(N_{1}, N_{0}\right)$ are index pairs for $S$ and $A$, respectively, and $\left(N_{2}, N_{1}\right)$ is a regular index pair for $A^{*}$. Furthermore, let $\iota: N_{1} / N_{0} \rightarrow N_{2} / N_{0}$ and $\pi: N_{2} / N_{0} \rightarrow N_{2} / N_{1}$ be the natural maps and let the connection map $\delta: N_{2} / N_{1} \rightarrow \Sigma N_{1} / N_{0}$ be defined by (5.9) and (5.6). For any $\lambda \in \Lambda$ let the corresponding maps be denoted by $\iota(\lambda): N_{1}(\lambda) / N_{0}(\lambda) \rightarrow N_{2}(\lambda) / N_{0}(\lambda), \pi(\lambda): N_{2}(\lambda) / N_{0}(\lambda) \rightarrow N_{2}(\lambda) / N_{1}(\lambda)$, $\delta(\lambda): N_{2}(\lambda) / N_{1}(\lambda) \rightarrow \Sigma N_{1}(\lambda) / N_{0}(\lambda)$. Then the diagram

$$
\begin{array}{cccc}
N_{1} / N_{0} \longrightarrow & \iota \\
\uparrow \iota(\lambda) & N_{2} / N_{0} \stackrel{\pi}{\longrightarrow} N_{2} / N_{1} \stackrel{\delta}{\longrightarrow} \Sigma N_{1} / N_{0} \\
\uparrow j(\lambda) & \uparrow k(\lambda) & \uparrow i(\lambda) \\
N_{1}(\lambda) / N_{0}(\lambda) \stackrel{\iota(\lambda)}{\longrightarrow} N_{2}(\lambda) / N_{0}(\lambda) \stackrel{\pi(\lambda)}{\longrightarrow} N_{2}(\lambda) / N_{1}(\lambda) \stackrel{\delta(\lambda)}{\longrightarrow} \Sigma N_{1}(\lambda) / N_{0}(\lambda)
\end{array}
$$

commutes, where $i(\lambda), j(\lambda)$ and $k(\lambda)$ are the natural inclusion maps. It follows from Theorem 5.7 and Proposition 6.5 that all the maps in diagram (6.23) induce morphisms of the corresponding connected simple systems which are independent of the choice of the index filtration $N_{0} \subset N_{1} \subset N_{2}$. Therefore we obtain the following commuting diagram of connected simple systems in which the rows are coexact. (6.24)

$$
\begin{aligned}
& I(A(K)) \stackrel{\iota}{\longrightarrow} I(S(K)) \stackrel{\pi}{\longrightarrow} I\left(A^{*}(K)\right) \stackrel{\delta}{\longrightarrow} \Sigma I(A(K)) \longrightarrow \\
& \quad \uparrow i(\lambda) \\
& I(\alpha(\lambda)) \stackrel{\iota(\lambda)}{\longrightarrow} I(\sigma(\lambda)) \stackrel{\pi(\lambda)}{\longrightarrow} I\left(\alpha^{*}(\lambda)\right) \stackrel{\delta(\lambda)}{\longrightarrow} \Sigma I(\alpha(\lambda)) \longrightarrow
\end{aligned}
$$

Here we have replaced $\Lambda$ by any compact subset $K \subset \Lambda$. If this set is contractible and satisfies the requirements of Lemma 6.6 for each of the index pairs $\left(N_{1}, N_{0}\right)$, $\left(N_{2}, N_{0}\right),\left(N_{2}, N_{1}\right)$, then it follows from Theorem 6.7 that the vertical maps in diagram (6.24) are equivalences for every $\lambda \in K$. The homotopy inverses of these equivalences define, of course, again morphisms between the respective connected simple systems and make the (vertically reverse) diagram commute. This implies that for any two points $\lambda, \mu$ in the same connected component of $\Lambda$ and any connecting sequence of compact contractible sets $K_{j} \subset \Lambda$ which satisfy the conditions of Lemma 6.6 there is a (unique) commuting diagram of the form (6.25)

$$
\begin{aligned}
& I(\alpha(\mu)) \stackrel{\iota(\mu)}{\longrightarrow} I(\sigma(\mu)) \stackrel{\pi(\mu)}{\longrightarrow} I\left(\alpha^{*}(\mu)\right) \stackrel{\delta(\mu)}{\longrightarrow} \Sigma I(\alpha(\mu)) \longrightarrow \\
& \downarrow F(\lambda, \mu) \quad \downarrow G(\lambda, \mu) \quad \downarrow H(\lambda, \mu) \quad \downarrow \Sigma F(\lambda, \mu) \\
& I(\alpha(\lambda)) \stackrel{\iota(\lambda)}{\longrightarrow} I(\sigma(\lambda)) \stackrel{\pi(\lambda)}{\longrightarrow} I\left(\alpha^{*}(\lambda)\right) \stackrel{\delta(\lambda)}{\longrightarrow} \Sigma I(\alpha(\lambda)) \longrightarrow
\end{aligned}
$$


where the vertical morphisms are equivalences. Finally, it follows from Theorem 6.9 that the vertical morphisms in (6.25) are independent of the choice of the connecting sequence $K_{j}$ if $\Lambda$ is simply connected. This proves the following result (compare Kurland [8]).

THEOREM 6.10. If $\Lambda$ is simply connected, then the maps $\iota(\lambda): I(\alpha(\lambda)) \rightarrow I(\sigma(\lambda))$, $\pi(\lambda): I(\sigma(\lambda)) \rightarrow I\left(\alpha^{*}(\lambda)\right), \delta(\lambda): I\left(\alpha^{*}(\lambda)\right) \rightarrow \Sigma I(\alpha(\lambda))$ of $\S 5$ induce the following coexact sequence of connected simple systems:

$$
I(\alpha, X, \Lambda) \stackrel{\iota}{\rightarrow} I(\sigma, X, \Lambda) \stackrel{\pi}{\rightarrow} I\left(\alpha^{*}, X, \Lambda\right) \stackrel{\delta}{\rightarrow} \Sigma I(\alpha, X, \Lambda) \rightarrow \cdots
$$

7. Conclusions. In this paper we have given complete and simplified proofs for most of the basic abstract results in the index theory of isolated invariant sets. Of course, there are many questions left open.

One of them is to develop a continuation theorem for flows on fibrations rather than product spaces $X \times \Lambda$. Another problem is the relation between the global Conley index $I(\sigma, X, \Lambda)$ and the Conley index $I(S, X \times \Lambda)$ of the global isolated invariant set $S=\bigcup_{\lambda \in \Lambda} \sigma(\lambda)$ in the parametrized flow $X \times \Lambda$. For example, there is the question whether $I(\sigma, X, \Lambda)$ is isomorphic to $I(S, X \times \Lambda)$ if $\Lambda$ is simply connected.

Several other questions have been indicated by Conley [3]. Among these there is the observation that information gets lost by collapsing the exit set $N_{0}$ in the index pair $\left(N_{1}, N_{0}\right)$. This leads to the question whether a sequence of index pairs which collapse to $S$ gives more information which can be used in a nice way for the definition of algebraic invariants. Another possible refinement of the Conley index might be to consider only special classes of homotopies since all the maps and homotopies in the theory are given by flow induced maps.

Furthermore, there is a duality in homotopy theory between fibrations (mapping fibration, loop functor, exact sequence) and cofibrations (mapping cone, suspension functor, coexact sequence). A very nice presentation of these duality relations can be found in Whitehead [13]. Since index pairs only give rise to a coexact sequence there arises the question whether there is some kind of a dual concept.

Of course, there is a big area of open questions when it comes to the point of applying the index theory to obtain results for concrete differential equations. Despite the fact that the Conley index has proven to be a very useful tool for many problems, there is the question under which conditions infinite dimensional systems can be formulated in the framework of §3. For some cases this has been done, e.g. by Smoller [11]. If this is not possible then there arises the question what one can do if $X$ is not locally compact and $\Gamma$ is only a semiflow. Some steps in this direction have been taken by Rybakowski and Zehnder [10]. Another possibility might be to go to finite dimensional approximations (see for example Conley and Zehnder [4]).

We stop at this place since the list of open questions has no end.

Acknowledgement. The author wishes to thank Professor C. C. Conley for many helpful discussions and for his encouragement to write this paper. 


\section{REFERENCES}

1. N. P. Bhatia and G. P. Szegö, Stability theory of dynamical systems, Springer, Berlin, 1970.

2. G. D. Birkhoff, Dynamical systems, Amer. Math. Soc. Colloq. Publ., vol. 9, Amer. Math. Soc., Providence, R. I., 1927.

3. C. C. Conley, Isolated invariant sets and the Morse index, CBMS Regional Conf. Ser. in Math., no. 38, Amer. Math. Soc., Providence, R. I., 1976.

4. C. C. Conley and R. Zehnder, Morse type index theory for flows and periodic solutions for Hamiltonian systems, Mathematics Research Center, University of Wisconsin-Madison, TSR \#2567, 1983; Comm. Pure Appl. Math. 37 (1984).

5. R. Franzosa, Index filtrations and connection matrices for partially ordered Morse decompositions, $\mathrm{Ph}$. D. Thesis, University of Wisconsin-Madison, 1984.

6. H. L. Kurland, The Morse index of an isolated invariant set is a connected simple system, J. Differential Equations 42 (1981), 234-259.

7. __ Homotopy invariants of repeller-attractor pairs. I. The Puppe sequence of an R-A pair, J. Differential Equations 46 (1982), 1-31.

8. _ Homotopy invariants of repeller-attractor pairs. II. Continuation of an $R-A$ pair, J. Differential Equations 49 (1983), 281-329.

9. J. T. Montgomery, Cohomology of isolated invariant sets under perturbation, J. Differential Equations 13 (1973), 257-299.

10. K. P. Rybakowski and E. Zehnder, A Morse equation in Conley's index theory for semiflows on metric spaces, Institut fur Mathematik, Ruhr Universität Bochum, 1982.

11. J. A. Smoller, Shock waves and reaction diffusion equations, Springer-Verlag, New York, 1983.

12. E. H. Spanier, Algebraic topology, Springer-Verlag, New York, 1966.

13. G. W. Whitehead, Elements of homotopy theory, Springer-Verlag, New York, 1978.

Mathematics Research Center, University of Wisconsin, Madison, Wisconsin 53706

Current address: Forschungsinstitut Für Mathematik, ETH-Zentrum, CH-8092 Zürich, Switzerland 